Yuji Liu

\title{
Solvability of impulsive periodic boundary value problems for higher order fractional differential equations
}

Received: 28 January 2016 / Accepted: 20 July 2016 / Published online: 2 August 2016

(C) The Author(s) 2016. This article is published with open access at Springerlink.com

\begin{abstract}
A class of periodic boundary value problems for higher order fractional differential equations with impulse effects is considered. We first convert the problem to an equivalent integral equation. Then, using a fixed-point theorem in Banach space, we establish existence results of solutions for this kind of boundary value problem for impulsive singular higher order fractional differential equations. Two examples are presented to illustrate the efficiency of the results obtained.
\end{abstract}

Mathematics Subject Classification 34K37 $\cdot 34 \mathrm{~K} 45 \cdot 34 \mathrm{~B} 37 \cdot 34 \mathrm{~B} 15 \cdot 34 \mathrm{~B} 10 \cdot 92 \mathrm{D} 25 \cdot 34 \mathrm{~A} 37 \cdot 34 \mathrm{~K} 15$

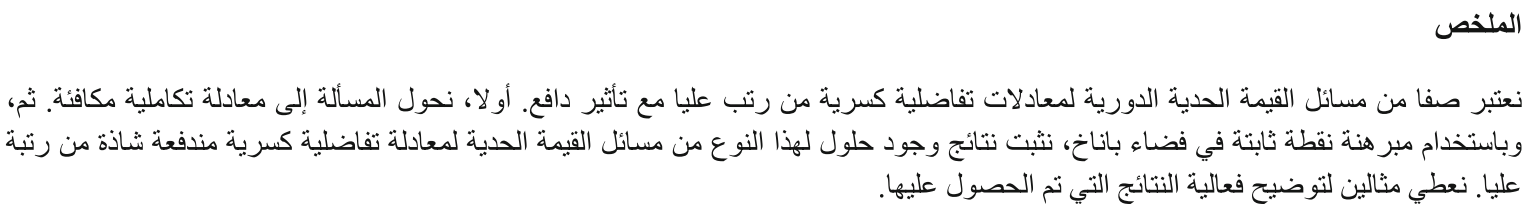

\section{Introduction}

Fractional differential equation is a generalization of ordinary differential equation to arbitrary non-integer orders. Fractional differential equations, therefore, find numerous applications in different branches of physics, chemistry, and biological sciences, such as visco-elasticity, feed back amplifiers, electrical circuits, electro analytical chemistry, fractional multipoles, and neuron modelling. The reader may refer to the books and monographs $[1,2,7,9]$ for fractional calculus and developments on fractional differential and fractional integrodifferential equations with applications.

On the other hand, the theory of impulsive differential equations describes processes which experience a sudden change of their state at certain moments. Processes with such characteristics arise naturally and often, for example, in phenomena studied in physics, chemical technology, population dynamics, biotechnology, and economics. For an introduction of the basic theory of impulsive differential equations, we refer the reader to $[3,4]$.

Some authors have addressed the solvability of boundary value problems for impulsive fractional differential equations, see $[8,10-12,15-17]$, but the existence of solutions of boundary value problems for higher order impulsive fractional differential equations has not been well studied. The reason is that it is difficult to transform a boundary value problem for higher order impulsive fractional differential equations to integral equations.

Y. Liu $(\varangle)$

Department of Mathematics, Guangdong University of Finance and Economics, Guangzhou 510320, People's Republic of China E-mail: liuyuji888@sohu.com 
In [18], the authors studied the existence of positive solutions of the following non-linear impulsive fractional differential equation with generalized periodic boundary value conditions:

$$
\left\{\begin{array}{l}
{ }^{c} D_{0^{+}}^{q} u(t)=f(t, u(t)), t \in J^{\prime}=J \backslash\left\{t_{1}, t_{2}, \ldots, t_{m}\right\}, J=[0,1], \\
\Delta u\left(t_{k}\right)=I_{k}\left(u\left(t_{k}\right)\right), \Delta u^{\prime}\left(t_{k}\right)=J_{k}\left(u\left(t_{k}\right)\right), k=1,2, \ldots, m, \\
a u(0)-b u(1)=a u^{\prime}(0)-b u^{\prime}(1)=0,
\end{array}\right.
$$

where $a$ and $b$ are real constants with $a>b>0,{ }^{c} D_{0^{+}}^{q}$ is the Caputo fractional derivative of order $q \in(1,2)$, $f: J \times \mathbb{R} \rightarrow \mathbb{R}$ is jointly continuous, $I_{k}, J_{k} \in C([0, \infty),[0, \infty))$, the impulsive points are $0=t_{0}<$ $t_{1}<t_{2}<\cdots<t_{m}<t_{m+1}=1, \Delta u\left(t_{k}\right)=\lim _{h \rightarrow 0^{+}} u\left(t_{k}+h\right)-\lim _{h \rightarrow 0^{+}} u\left(t_{k}-h\right)$, and $\Delta u^{\prime}\left(t_{k}\right)=$ $\lim _{h \rightarrow 0^{+}} u^{\prime}\left(t_{k}+h\right)-\lim _{h \rightarrow 0^{+}} u^{\prime}\left(t_{k}-h\right)$. The methods used in [18] are based upon the non-linear alternative of the Schauder and Guo-Krasnosel'skii fixed-point theorem on cones. We note that BVP (1.1) is a non-resonant problem under the assumption $a>b>0$.

In [14], the existence of solutions of a high-order impulsive boundary value problem for quasi-linear fractional differential equations was studied. Theorem 1 in [14] is as follows:

Result 1. Suppose that $n \geq 2$ is an integer, $q \in(n-1, n], \xi_{i} \in(0,1), y_{0}, y_{1} \in \mathbb{R}, b_{i} \geq 0$ with $\sum_{i=1}^{p} b_{i}<1$, $z \in C[0,1]$. Then

$$
\left\{\begin{array}{l}
{ }^{c} D_{0^{+}}^{q} y(t)+z(t)=0, t \in J^{\prime}=J \backslash\left\{t_{1}, t_{2}, \ldots, t_{p}\right\}, J=[0,1] \\
\Delta y\left(t_{k}\right)=I_{k}\left(y\left(t_{k}\right)\right), \Delta u^{(n-1)}\left(t_{k}\right)=J_{k}\left(y\left(t_{k}\right)\right), k=1, \ldots, p \\
\Delta y^{(i)}\left(t_{k}\right)=0, i=1, \ldots, n-2, k=1, \ldots, p \\
y^{(i)}(0)=0, i=1,2, \ldots, n-2, y(0)=y_{0}+g(y), y^{(n-1)}(1)=y_{1}+\sum_{i=1}^{m-2} b_{i} y^{(n-1)}\left(\xi_{i}\right)
\end{array}\right.
$$

has a unique solution

$$
y(t)=\left\{\begin{array}{l}
-\int_{0}^{t} \frac{(t-s)^{q-1}}{\Gamma(q)} z(s) \mathrm{d} s+c_{1}+\frac{c_{n-1}}{(n-1) !} t^{n-1}, t \in J_{0}=\left[0, t_{1}\right] \\
-\int_{t_{1}}^{t} \frac{(t-s)^{q-1}}{\Gamma(q)} z(s) \mathrm{d} s-\int_{0}^{t_{1}} \frac{\left(t_{1}-s\right)^{q-1}}{\Gamma(q)} z(s) \mathrm{d} s-\frac{\left(t-t_{1}\right)^{n-1}}{(n-1) !} \int_{0}^{t_{1}} \frac{\left(t_{1}-s\right)^{q-n}}{\Gamma(q-n+1)} z(s) \mathrm{d} s \\
+\frac{\left(t-t_{1}\right)^{n-1}}{(n-1) !} J_{1}\left(y\left(t_{1}\right)\right)+I_{1}\left(y\left(t_{1}\right)\right)+c_{1}+\frac{c_{n-1}}{(n-1) !}\left[t_{1}^{n-1}+\left(t-t_{1}\right)^{n-1}\right], t \in J_{1}=\left(t_{1}, t_{2}\right], \\
-\int_{t_{k}}^{t} \frac{(t-s)^{q-1}}{\Gamma(q)} z(s) \mathrm{d} s-\sum_{i=1}^{k} \int_{t_{i-1}}^{t_{i}} \frac{\left(t_{i}-s\right)^{q-1}}{\Gamma(q)} z(s) \mathrm{d} s-\frac{\left(t-t_{k}\right)^{n-1}}{(n-1) !} \sum_{i=1}^{k} \int_{t_{i-1}}^{t_{i}} \frac{\left(t_{i}-s\right)^{q-n}}{\Gamma(q-n+1)} z(s) \mathrm{d} s \\
+\frac{\sum_{i=2}^{k} \sum_{j=i-1}^{k-1}\left(t_{j+1}-t_{j}\right)^{n-1}}{(n-1) !} \int_{t_{i-2}}^{t_{i-1}} \frac{\left(t_{i-1}-s\right)^{q-n}}{\Gamma(q-n+1)} z(s) \mathrm{d} s \\
+\frac{\left(t-t_{k}\right)^{n-1}}{(n-1) !} \sum_{i=1}^{k} J_{i}\left(y\left(t_{i}\right)\right)+\frac{\sum_{i=1}^{k-1} \sum_{j=i}^{k-1}\left(t_{j+1}-t_{j}\right)^{n-1}}{(n-1) !} J_{i}\left(y\left(t_{i}\right)\right)+\sum_{i=1}^{k} I_{i}\left(y\left(t_{i}\right)\right) \\
+c_{1}+\frac{c_{n-1}}{(n-1) !}\left[\sum_{i=1}^{k}\left(t_{i}-t_{i-1}\right)^{n-1}+\left(t-t_{k}\right)^{n-1}\right], t \in J_{k}=\left(t_{k}, t_{k+1}\right], k=2,3, \ldots, p,
\end{array}\right.
$$

where

$$
\begin{aligned}
c_{1}= & y_{0}+g(y), d=\sum_{i=1}^{m-2} b_{i}, \\
c_{n-1}= & \frac{1}{1-d} \int_{t_{p}}^{1} \frac{(1-s)^{q-n}}{\Gamma(q-n+1)} z(s) \mathrm{d} s+\sum_{i=1}^{p} \int_{t_{i-1}}^{t_{i}} \frac{\left(t_{i}-s\right) 6 q-n}{\Gamma(q-n+1)} z(s) \mathrm{d} s \\
& +\frac{1}{1-d} \sum_{j=1}^{m-2} b_{j} \int_{t_{p}}^{\xi_{j}} \frac{\left(\xi_{j}-s\right)^{q-n}}{\Gamma(q-n+1)} z(s) \mathrm{d} s-\sum_{i=1}^{p} J_{i}\left(y\left(t_{i}\right)\right)+\frac{y_{1}}{1-d} .
\end{aligned}
$$


We find that Result 1 is wrong. In fact, consider the following problem:

$$
\left\{\begin{array}{l}
{ }^{c} D_{0^{+}}^{q} y(t)+z(t)=0, t \in J^{\prime}=J \backslash\left\{t_{1}, t_{2}, \ldots, t_{p}\right\}, J=[0,1], \\
\Delta y\left(t_{k}\right)=I_{k}, \Delta u^{\prime}\left(t_{k}\right)=J_{k}, k=1, \ldots, p, \\
y(0)=y_{0}, y^{\prime}(1)=y_{1},
\end{array}\right.
$$

where $q \in(1,2), 0=t_{0}<t_{1}<\cdots<t_{p}<t_{p+1}=1, y_{0}, y_{1} \in \mathbb{R}, I_{k}, J_{k} \in \mathbb{R}(k=1,2, \ldots, p)$. By Lemma 2.11 in Sect. 2, there exist constants $c_{k}, d_{k} \in \mathbb{R}$, such that

$$
y(t)=\sum_{j=0}^{i} c_{j}+\sum_{j=0}^{i} d_{j}\left(t-t_{j}\right)-\int_{0}^{t} \frac{(t-s)^{q-1}}{\Gamma(q)} z(s) \mathrm{d} s, \quad t \in\left(t_{i}, t_{i+1}\right], i=0,1, \ldots, p .
$$

By $y(0)=y_{0}$, we get $c_{0}=y_{0}$. By $\Delta y\left(t_{k}\right)=I_{k}$, we get $c_{k}=I_{k}(k=1,2, \ldots, p)$. By $\Delta u^{\prime}\left(t_{k}\right)=J_{k}$, we get $d_{k}=J_{k}(k=1,2, \ldots, p)$. From $y^{\prime}(1)=y_{1}$, we get $\sum_{j=0}^{p} d_{j}-\int_{0}^{1} \frac{(1-s)^{q-2}}{\Gamma(q-1)} z(s) \mathrm{d} s=y_{1}$. Then, $d_{0}=y_{1}+\sum_{j=1}^{i} J_{j}+\int_{0}^{1} \frac{(1-s)^{q-2}}{\Gamma(q-1)} z(s) \mathrm{d} s$. Hence

$$
\begin{aligned}
y(t)= & y_{0}+t\left(y_{1}+\sum_{j=1}^{i} J_{j}+\int_{0}^{1} \frac{(1-s)^{q-2}}{\Gamma(q-1)} z(s) \mathrm{d} s\right) \\
& +\sum_{j=1}^{i} I_{j}+\sum_{j=0}^{i} J_{j}\left(t-t_{j}\right)-\int_{0}^{t} \frac{(t-s)^{q-1}}{\Gamma(q)} z(s) \mathrm{d} s, \quad t \in\left(t_{i}, t_{i+1}\right], \quad i=0,1, \ldots, p .
\end{aligned}
$$

It is easy to see that (1.5) is different from (1.3). By direct computation, we know that (1.5) satisfies (1.4). Therefore, Result 1 is wrong. The reason of this mistake comes from the transformation from (1.2) to (1.3). Hence, it is interesting to find a new method for converting a BVP for impulsive fractional differential equations to an integral equation.

Motivated by [14], in this paper, we discuss the following periodic boundary value problems for non-linear impulsive singular fractional differential equation:

$$
\begin{cases}{ }^{c} D_{0^{+}}^{\alpha} u(t)=f\left(t, u(t),{ }^{c} D_{0^{+}}^{\beta} u(t)\right), & t \in\left(t_{s}, t_{s+1}\right], s \in \mathbb{N}_{0}^{m}, \\ u^{(j)}(0)=u^{(j)}(1), & j \in \mathbb{N}_{0}^{n-1}, \\ \Delta u^{(j)}\left(t_{s}\right)=I_{j}\left(t_{s}, u\left(t_{s}\right),{ }^{c} D_{0^{+}}^{\beta} u\left(t_{s}\right)\right), & j \in \mathbb{N}_{0}^{n-1}, s \in \mathbb{N}_{1}^{m},\end{cases}
$$

where

(a) $\beta \in(0, \alpha-n+1), n-1<\alpha<n, n$ is a positive integer, ${ }^{c} D_{0^{+}}^{*}$ is the Caputo fractional derivative of orders $*$ with starting point 0 ,

(b) $0=t_{0}<t_{1}<\cdots<t_{s}<\cdots<t_{m}<t_{m+1}=1$ with $m$ being a positive integer, $\mathbb{N}_{a}^{b}=\{a, a+1, a+$ $2, \ldots, b\}$ for non-negative integers $a<b$, and

(c) $f:(0,1) \times \mathbb{R}^{2} \rightarrow \mathbb{R}, I_{j}:\left\{t_{s}: s \in \mathbb{N}_{1}^{m}\right\} \times \mathbb{R}^{2} \rightarrow \mathbb{R}, f$ are Carathéodory functions, $I_{j}\left(j \in \mathbb{N}_{1}^{m}\right)$ are discrete Carathéodory functions.

A function $x:(0,1] \rightarrow \mathbb{R}$ is said to be a solution of (1.7) if

$$
\begin{aligned}
& \left.x\right|_{\left(t_{s}, t_{s+1}\right]},\left.{ }^{c} D_{0^{+}}^{\beta} x\right|_{\left(t_{s}, t_{s+1}\right]} \in C^{0}\left(t_{s}, t_{s+1}\right], s \in N_{0}^{m}, \\
& \lim _{t \rightarrow t_{s}^{+}} x(t), \lim _{t \rightarrow t_{s}^{+}}{ }^{c} D_{0^{+}}^{\beta} x(t) \text { exist for all } s \in \mathbb{N}_{0}^{m}, \\
& { }^{c} D_{0^{+}}^{\alpha} x \text { is measurable on each }\left(t_{i}, t_{i+1}\right], i \in \mathbb{N}_{0}^{m}
\end{aligned}
$$

and $x$ satisfies all equations in (1.7). 
The first purpose of this paper is to present a new method for converting BVPs for impulsive fractional differential equation to integral equations, see Lemma 2.11 in Sect. 2. The second purpose is to construct a weighted Banach space and to apply the Leray-Schauder non-linear alternative to establish some existence results of at least one solution of (1.7). Our results are new and naturally complement the literature on impulsive fractional differential equations.

The paper is outlined as follows. Section 2 contains some preliminary results. Main results are presented in Sect. 3. Finally, in Sect. 4, we give an example to illustrate the efficiency of the results obtained.

\section{Preliminaries}

For the convenience of the readers, we shall state the necessary definitions from fractional calculus theory.

For $\phi \in L^{1}(0, \infty)$, denote $\|\phi\|_{1}=\int_{0}^{\infty}|\phi(s)| \mathrm{d} s$. Let the Gamma and Beta functions $\Gamma(\alpha)$ and $\mathbf{B}(p, q)$ be defined by

$$
\Gamma(\alpha)=\int_{0}^{+\infty} x^{\alpha-1} e^{-x} \mathrm{~d} x, \quad \mathbf{B}(p, q)=\int_{0}^{1} x^{p-1}(1-x)^{q-1} \mathrm{~d} x
$$

Definition 2.1 [9] The Riemann-Liouville fractional integral of order $\alpha>0$ of a function $g:(0, \infty) \rightarrow \mathbb{R}$ is given by

$$
I_{0^{+}}^{\alpha} g(t)=\frac{1}{\Gamma(\alpha)} \int_{0}^{t}(t-s)^{\alpha-1} g(s) \mathrm{d} s,
$$

provided that the right-hand side exists.

Definition 2.2 [9] The Caputo fractional derivative of order $\alpha>0$ of a continuous function $g:(0, \infty) \rightarrow \mathbb{R}$ is given by

$$
{ }^{c} D_{0^{+}}^{\alpha} g(t)=\frac{1}{\Gamma(n-\alpha)} \int_{0}^{t} \frac{g^{(n)}(s)}{(t-s)^{\alpha-n+1}} \mathrm{~d} s,
$$

where $n-1 \leq \alpha<n$, provided that the right-hand side exists.

Definition 2.3 Let $p>-1, q \in(-1,0]$. We say $K:(0,1) \times \mathbb{R}^{4} \rightarrow \mathbb{R}$ is a Carathéodory function if it satisfies the followings:

(i) $t \rightarrow K(t, x, y)$ is integral on $\left(t_{s}, t_{s+1}\right]$ for every $(x, y) \in \mathbb{R}^{2}, s \in \mathbb{N}_{0}^{m}$,

(ii) $(x, y) \rightarrow K(t, x, y)$ is continuous on $\mathbb{R}^{2}$ for all $t \in\left(t_{s}, t_{s+1}\right]\left(s \in \mathbb{N}_{0}^{m}\right)$; and

(iii) for each $r>0$ there exists a constant $A_{r, f} \geq 0$, such that

$$
|K(t, x, y)| \leq A_{r, K} t^{p}(1-t)^{q}
$$

holds for $t \in\left(t_{s}, t_{s+1}\right], s \in \mathbb{N}_{0}^{m},|x|,|y| \leq r$.

Definition 2.4 $G:\left\{t_{s}: s \in \mathbb{N}_{1}^{m}\right\} \times \mathbb{R}^{2} \rightarrow \mathbb{R}$ is called a discrete Carathéodory function if

(i) $(x, y) \rightarrow G\left(t_{s}, x, y\right)$ is continuous on $\mathbb{R}^{2}$ for each $s \in \mathbb{N}_{1}^{m}$,

(ii) for each $r>0$ there exists $A_{r, G, s} \geq 0$, such that

$$
\left|G\left(t_{s}, x, y\right)\right| \leq A_{r, G, s}
$$

holds for $|x|,|y| \leq r, s \in \mathbb{N}_{1}^{m}$.

Definition 2.5 [6] Let $E$ and $Z$ be Banach spaces. $L: D(L) \subset E \rightarrow Z$ is called a Fredholm operator of index zero if $\operatorname{Im} L$ is closed in $E$ and $\operatorname{dim} \operatorname{Ker} L=\operatorname{codim} \operatorname{Im} L<+\infty$.

It is easy to see that there exist the projectors $P: E \rightarrow E$, and $Q: Z \rightarrow Z$, such that

$$
\operatorname{Im} P=\operatorname{Ker} L, \operatorname{Ker} Q=\operatorname{Im} L, \quad X=\operatorname{Ker} L \oplus \operatorname{Ker} P, \quad Y=\operatorname{Im} L \oplus \operatorname{Im} Q
$$

if $L$ is a Fredholm operator of index zero.

If $L: D(L) \subset E \rightarrow Z$ is called a Fredholm operator of index zero, the inverse of

$$
\left.L\right|_{D(L) \cap \operatorname{Ker} P}: D(L) \cap \operatorname{Ker} P \rightarrow \operatorname{Im} L
$$

is denoted by $K_{p}$. 
Definition 2.6 [6] Suppose that $L: D(L) \subset E \rightarrow Z$ is a Fredholm operator of index zero. For non-empty open bounded subset $\Omega$ of $E$ satisfying $D(L) \cap \bar{\Omega} \neq \emptyset$, the continuous map $N: \bar{\Omega} \rightarrow Z$ is called $L$-compact if $Q N(\bar{\Omega})$ is bounded and $K_{p}(I-Q) N(\bar{\Omega})$ is bounded and relatively compact.

To obtain the main results, we need the Leray-Schauder non-linear alternative.

Lemma 2.7 [6] Let $L$ be a Fredholm operator of index zero and $N$ be L compact on each open non-empty set $\Omega$ centered at zero. Assume that the following conditions are satisfied:

(i) $L x \neq \lambda N x$ for every $(x, \lambda) \in[D(L) \backslash \operatorname{Ker} L) \cap \partial \Omega] \times(0,1)$;

(ii) $N x \notin \operatorname{ImL}$ for every $x \in \operatorname{Ker} L \cap \partial \Omega$; and

(iii) $\operatorname{deg}\left(\left.\wedge^{-1} Q N\right|_{\text {KerL }}, \Omega \cap \operatorname{KerL}, 0\right) \neq 0$, where $\wedge^{-1}: Y / \operatorname{ImL} \rightarrow$ KerL is the inverse of the isomorphism $\wedge: \operatorname{Ker} L \rightarrow Y / \operatorname{ImL}$.

Then, the equation $L x=N x$ has at least one solution in $D(L) \cap \bar{\Omega}$.

Lemma 2.8 (Lemma 2.22 in [2]) Let $n$ be a positive integer. Suppose that $\alpha \in(n-1, n), h \in L^{1}\left(0, t_{1}\right) \bigcap$ $C^{0}\left(0, t_{1}\right)$. Then, $x$ is a solution of ${ }^{c} D_{0^{+}}^{\alpha} x(t)=h(t)$, a.e., $t \in\left(0, t_{1}\right]$ if and only if there exist constants $c_{v 0} \in \mathbb{R}$, such that

$$
x(t)=\sum_{v=0}^{n-1} \frac{c_{v 0}}{\Gamma(v+1)} t^{v}+\int_{0}^{t} \frac{(t-s)^{\alpha-1}}{\Gamma(\alpha)} h(s) \mathrm{d} s, t \in\left(0, t_{1}\right] .
$$

Lemma 2.9 Suppose that $h$ is integral on each subinterval of $(0,1)$. Then, $x$ satisfying

$$
\begin{aligned}
& \left.x^{(j)}\right|_{\left(t_{s}, t_{s+1}\right]} \in C^{0}\left(t_{s}, t_{s+1}\right], \quad s \in \mathbb{N}_{0}, j \in \mathbb{N}_{0}^{n-1}, \\
& \lim _{t \rightarrow t_{s}^{+}} x^{(j)}(t) \text { exists for all } s \in \mathbb{N}_{0}, j \in \mathbb{N}_{0}^{n-1}
\end{aligned}
$$

is a solution of

$$
{ }^{c} D_{0^{+}}^{\alpha} x(t)=h(t), \text { a.e., } t \in\left(t_{i}, t_{i+1}\right]\left(i \in \mathbb{N}_{0}^{m}\right)
$$

if and only if there exist constants $c_{v 0} \in \mathbb{R}$, such that

$$
x(t)=\sum_{j=0}^{i} \sum_{v=0}^{n-1} \frac{c_{v j}}{\Gamma(v+1)}\left(t-t_{j}\right)^{v}+\int_{0}^{t} \frac{(t-s)^{\alpha-1}}{\Gamma(\alpha)} h(s) \mathrm{d} s, \quad t \in\left(t_{i}, t_{i+1}\right], \quad i \in \mathbb{N}_{0}^{m} .
$$

Proof By Lemma 2.7, we know that $x$ satisfying (2.1) is a solution of (2.2) if and only if $x$ satisfies (2.3) on $\left(t_{0}, t_{1}\right]$. To complete the proof, we consider two steps:

Step 1 We prove that $x$ satisfies (2.1) and (2.2) if $x$ satisfies (2.3).

From (2.3), we obviously know that (2.1) holds. We need to prove that (2.2) holds on all $\left(t_{i}, t_{i+1}\right]\left(i \in \mathbb{N}_{0}^{m}\right)$. In fact, for $t \in\left(t_{0}, t_{1}\right]$, by Lemma 2.7, we know $D_{0^{+}}^{\alpha} x(t)=h(t)$. For $t \in\left(t_{i}, t_{i+1}\right]$, we have

$$
\begin{aligned}
{ }^{c} D_{0^{+}}^{\alpha} x(t)= & \frac{1}{\Gamma(n-\alpha)} \int_{0}^{t}(t-s)^{n-\alpha-1} x^{(n)}(s) \mathrm{d} s \\
= & \frac{\sum_{j=0}^{i-1} \int_{t_{j}}^{t_{j+1}}(t-s)^{n-\alpha-1} x^{(n)}(s) \mathrm{d} s+\int_{t_{i}}^{t}(t-s)^{n-\alpha-1} x^{(n)}(s) \mathrm{d} s}{\Gamma(n-\alpha)} \\
= & \frac{\sum_{j=0}^{i-1} \int_{t_{j}}^{t_{j+1}}(t-s)^{n-\alpha-1}\left(\sum_{v=0}^{j} \sum_{\kappa=0}^{n-1} \frac{c_{\kappa v}}{\Gamma(\kappa+1)}\left(s-t_{v}\right)^{\kappa}+\int_{0}^{s} \frac{(s-u)^{\alpha-1}}{\Gamma(\alpha)} h(u) \mathrm{d} u\right)^{(n)} \mathrm{d} s}{\Gamma(n-\alpha)} \\
& +\frac{\int_{t_{i}}^{t}(t-s)^{n-\alpha-1}\left(\sum_{v=0}^{i} \sum_{\kappa=0}^{n-1} \frac{c_{\kappa v}}{\Gamma(\kappa+1)}\left(s-t_{v}\right)^{\kappa}+\int_{0}^{s} \frac{(s-u)^{\alpha-1}}{\Gamma(\alpha)} f(u) \mathrm{d} u\right)^{(n)} \mathrm{d} s}{\Gamma(n-\alpha)}
\end{aligned}
$$




$$
\begin{aligned}
& =\frac{\sum_{j=0}^{i-1} \int_{t_{j}}^{t_{j+1}}(t-s)^{n-\alpha-1}\left(\int_{0}^{s} \frac{(s-u)^{\alpha-1}}{\Gamma(\alpha)} h(u) \mathrm{d} u\right)^{(n)} \mathrm{d} s}{\Gamma(n-\alpha)}+\frac{\int_{t_{i}}^{t}(t-s)^{n-\alpha-1}\left(\int_{0}^{s} \frac{(s-u)^{\alpha-1}}{\Gamma(\alpha)} f(u) \mathrm{d} u\right)^{(n)} \mathrm{d} s}{\Gamma(n-\alpha)} \\
& =\frac{\int_{0}^{t}(t-s)^{n-\alpha-1}\left(\int_{0}^{s} \frac{(s-u)^{\alpha-n}}{\Gamma(\alpha-n+1)} h(u) \mathrm{d} u\right)^{\prime} \mathrm{d} s}{\Gamma(n-\alpha)} \\
& =\left[\frac{\int_{0}^{t}(t-s)^{n-\alpha}\left(\int_{0}^{s} \frac{(s-u)^{\alpha-n}}{\Gamma(\alpha-n+1)} h(u) \mathrm{d} u\right)^{\prime} \mathrm{d} s}{(n-\alpha) \Gamma(n-\alpha)}\right]^{\prime} \\
& =\left[\frac{\left.(t-s)^{n-\alpha} \int_{0}^{s} \frac{(s-u)^{\alpha-n}}{\Gamma(\alpha-n+1)} h(u) \mathrm{d} u\right|_{0} ^{t}+(n-\alpha) \int_{0}^{t}(t-s)^{n-\alpha-1} \int_{0}^{s} \frac{(s-u)^{\alpha-n}}{\Gamma(\alpha-n+1)} h(u) \mathrm{d} u \mathrm{~d} s}{(n-\alpha) \Gamma(n-\alpha)}\right]^{\prime} \\
& =\left[\frac{\int_{0}^{t}(t-s)^{n-\alpha-1} \int_{0}^{s} \frac{(s-u)^{\alpha-n}}{\Gamma(\alpha-n+1)} h(u) \mathrm{d} u \mathrm{~d} s}{\Gamma(n-\alpha)}\right]^{\prime} \\
& =\left[\frac{\int_{0}^{t} \int_{s}^{t}(t-s)^{n-\alpha-1} \frac{(s-u)^{\alpha-n}}{\Gamma(\alpha-n+1)} \mathrm{d} s h(u) \mathrm{d} u}{\Gamma(n-\alpha)}\right]^{\prime}=\left[\frac{\int_{0}^{t} \int_{0}^{1}(1-w)^{n-\alpha-1} \frac{w^{\alpha-n}}{\Gamma(\alpha-n+1)} d w h(u) \mathrm{d} u}{\Gamma(n-\alpha)}\right]^{\prime} \\
& =h(t), t \in\left(t_{i}, t_{i+1}\right], \quad i \in \mathbb{N}_{1} .
\end{aligned}
$$

It follows that $x$ satisfies (2.2).

Step 2 We prove that $x$ satisfies (2.3) if $x$ satisfies (2.1) and (2.2). By Lemma 2.7, from (2.1) and (2.2), we know that (2.3) holds for $i=0$. We suppose that (2.3) holds for $0,1,2, \ldots, i$. We will prove that (2.3) holds for $i+1$. Then, by mathematical induction method, we see that (2.3) holds for all $i \in \mathbb{N}_{0}^{m}$.

In fact, we suppose that

$$
x(t)=\Phi(t)+\sum_{j=0}^{i} \sum_{v=0}^{n-1} \frac{c_{v j}}{\Gamma(v+1)}\left(t-t_{j}\right)^{v}+\int_{0}^{t} \frac{(t-s)^{\alpha-1}}{\Gamma(\alpha)} h(s) \mathrm{d} s, \quad t \in\left(t_{i+1}, t_{i+2}\right] .
$$

Then, for $t \in\left(t_{i+1}, t_{i+2}\right]$, we have

$$
\begin{aligned}
h(t)= & { }^{c} D_{0^{+}}^{\alpha} x(t)=\frac{1}{(n-\alpha)}\left[\sum_{j=0}^{i} \int_{t_{j}}^{t_{j+1}}(t-s)^{n-\alpha-1} x^{(n)}(s) \mathrm{d} s+\int_{t_{i+1}}^{t}(t-s)^{n-\alpha-1} x^{(n)}(s) \mathrm{d} s\right] \\
= & \frac{\sum_{j=0}^{i} \int_{t_{j}}^{t_{j+1}}(t-s)^{n-\alpha-1}\left(\sum_{\kappa=0}^{j} \sum_{v=0}^{n-1} \frac{c_{v \kappa}}{\Gamma(v+1)}\left(s-t_{\kappa}\right)^{v}+\int_{0}^{s} \frac{(s-v)^{\alpha-1}}{\Gamma(\alpha-1)} h(v) \mathrm{d} v\right)^{(n)} \mathrm{d} s}{\Gamma(n-\alpha)} \\
& +\frac{\int_{t_{i+1}}^{t}(t-s)^{n-\alpha-1}\left(\Phi(s)+\sum_{\kappa=0}^{k} \sum_{v=0}^{n-1} \frac{c_{v \kappa}}{\Gamma(\alpha-v+1)}\left(s-t_{\kappa}\right)^{v}+\int_{0}^{s} \frac{(s-v)^{\alpha-1}}{\Gamma(\alpha-1)} h(v) \mathrm{d} v\right)^{(n)} \mathrm{d} s}{\Gamma(n-\alpha)} \\
= & { }^{c} D_{t_{i+1}^{+}}^{\alpha} \Phi(t)+\frac{\int_{0}^{t}(t-s)^{n-\alpha-1}\left(\int_{0}^{s} \frac{(s-v)^{\alpha-1}}{\Gamma(\alpha)} h(v) \mathrm{d} v\right)^{(n)} \mathrm{d} s}{\Gamma(n-\alpha)} .
\end{aligned}
$$

Similarly to Step 1 , we can get that

$$
h(t)={ }^{c} D_{0^{+}}^{\alpha} x(t)=h(t)+{ }^{c} D_{t_{i+1}^{+}}^{\alpha} \Phi(t) .
$$

Therefore, ${ }^{c} D_{t_{i+1}^{+}}^{\alpha} \Phi(t)=0$ on $\left(t_{i+1}, t_{i+2}\right]$. Then, there exist constants $c_{v i+1} \in R\left(v \in \mathbb{N}_{0}^{n-1}\right)$, such that $\Phi(t)=\sum_{v=0}^{n} c_{v i+1} \frac{\left(t-t_{i+1}\right)^{v}}{\Gamma(v+1)}$ on $\left(t_{i+1}, t_{i+2}\right]$. Substituting $\Phi$ into (2.4), we get that (2.3) holds for $i+1$. By 
the mathematical induction method, we know that (2.3) holds for all $i \in \mathbb{N}_{0}^{m}$. Therefore, $x$ satisfies (2.3) if $x$ satisfies (2.1) and (2.2). The proof is complete.

Remark 2.10 Lemma 2.12 in [10] claimed that if $h \in P C[0,1], \mathbb{R})$ and $\alpha \in(n-1, n)$, then $y \in A^{n}[0,1]$ satisfies ${ }^{c} D_{0^{+}}^{\alpha} y(t)=h(t)$ if and only if $y \in P C^{n-1}[0,1]$ and

$$
y(t)=\sum_{j=0}^{n-1} \frac{y^{(j)}(0)}{(j !} t^{j}+\sum_{j=0}^{n-1}\left[\sum_{0<t_{k}<t} \frac{\Delta y^{(k)}\left(t_{k}\right)}{j !}\left(t-t_{k}\right)^{j}\right]+\int_{0}^{t} \frac{(t-s)^{\alpha-1}}{\Gamma(\alpha)} h(s) \mathrm{d} s, \quad t \in[0,1] .
$$

It is easy to see that Lemma 2.11 in this paper is similar to Lemma 2.12 in [10]. However, our proof is different from that in [10].

Define

$$
X=\left\{x:(0,1] \rightarrow \mathbb{R}: \begin{array}{c}
\left.x\right|_{\left(t_{s}, t_{s+1}\right]},\left.{ }^{c} D_{0^{+}}^{\beta} x\right|_{\left(t_{s}, t_{s+1}\right]} \in C^{0}\left(t_{s}, t_{s+1}\right]\left(s \in \mathbb{N}_{0}\right), \\
\lim _{t \rightarrow t_{s}^{+}} x(t), \lim _{t \rightarrow t_{s}^{+}}{ }^{c} D_{0^{+}}^{\beta} x(t) \text { exist }\left(s \in \mathbb{N}_{0}^{m}\right) .
\end{array}\right\}
$$

For $x \in X$, define the norms by

$$
\|x\|=\|x\|_{X}=\max \left\{\sup _{t \in(0,1]}|x(t)|, \sup _{t \in(0,1]}\left|{ }^{c} D_{0^{+}}^{\beta} x(t)\right|\right\} .
$$

Lemma 2.11 $X$ is a Banach space.

Proof The proof is standard and omitted.

Lemma 2.12 Let $M$ be a subset of $X$. Then, $M$ is relatively compact if and only if the following conditions are satisfied:

(i) both $\{t \rightarrow x(t): x \in M\}$ and $\left\{t \rightarrow D_{0^{+}}^{\beta} x(t): x \in M\right\}$ are uniformly bounded,

(ii) both $\{t \rightarrow x(t): x \in M\}$ and $\left\{t \rightarrow D_{0^{+}}^{\beta} x(t): x \in M\right\}$ are equicontinuous in any interval $\left(t_{s}, t_{s+1}\right](s \in$ $\left.\mathbb{N}_{0}\right)$.

Proof The proof is standard and omitted.

For $x \in X$, denote $f_{x}(t)=f\left(t, x(t),{ }^{c} D_{0^{+}}^{\beta} x(t)\right)$ and $I_{j x}\left(t_{s}\right)=I_{j}\left(t_{s}, x\left(t_{s}\right),{ }^{c} D_{0^{+}}^{\beta} x\left(t_{s}\right)\right)$. Choose $Z=$ $L^{1}(0,1) \times \mathbb{R}^{n m}$ with the norm $\left\|\left(x, a_{i j}: i \in \mathbb{N}_{0}^{n-1}, j \in \mathbb{N}_{1}^{m}\right)\right\|=\max \left\{\|x\|_{1},\left|a_{i j}\right|: i \in \mathbb{N}_{0}^{n-1}, j \in \mathbb{N}_{1}^{m}\right\}$. Choose $E=X$ and $D\left(L_{1}\right)=\left\{x \in X:{ }^{c} D_{0^{+}}^{\alpha} x \in L^{1}(0,1), x^{(i)}(0)=x^{(i)}(1), i \in \mathbb{N}_{0}^{n-1}\right\}$. Define the linear operator $L_{1}: X \cap D\left(L_{1}\right) \rightarrow Z$ and the non-linear operator $N_{1}: X \rightarrow Z$ by

$$
\left(L_{1} x\right)(t)=\left(\begin{array}{c}
{ }^{c} D_{0^{+}}^{\alpha} x(t) \\
\Delta x\left(t_{s}\right): s \in \mathbb{N}_{1}^{m} \\
\Delta x^{\prime}\left(t_{s}\right): s \in \mathbb{N}_{1}^{m} \\
\cdots \\
\Delta x^{(n-1)}\left(t_{s}\right): s \in \mathbb{N}_{1}^{m}
\end{array}\right), \quad\left(N_{1} x\right)(t)=\left(\begin{array}{c}
f_{x}(t) \\
I_{0 x}\left(t_{s}\right): s \in \mathbb{N}_{1}^{m} \\
I_{1 x}\left(t_{s}\right): s \in \mathbb{N}_{1}^{m} \\
\cdots \\
I_{n-1 x}\left(t_{s}\right): s \in \mathbb{N}_{1}^{m}
\end{array}\right) .
$$

Denote

$$
\bar{M}=\left(m_{i j}\right)_{(n-1) \times(n-1)}=\left(\begin{array}{ccccc}
\frac{1}{\Gamma(2)} & 0 & 0 & \cdots & 0 \\
\frac{1}{\Gamma(3)} & \frac{1}{\Gamma(2)} & 0 & \cdots & 0 \\
\cdots & \cdots & \cdots & \cdots & \cdots \\
\frac{1}{\Gamma(n)} & \frac{1}{\Gamma(n-1)} & \frac{1}{\Gamma(n-3)} & \cdots & \frac{1}{\Gamma(2)}
\end{array}\right) .
$$

Then, $|\bar{M}|=\left|m_{i, j}\right|_{(n-1) \times(n-1)}=1$. One has for $i, j \in \mathbb{N}_{1}^{n-1}$ that

$$
\left|m_{i, j}\right|_{(n-1) \times(n-1)}=\sum_{i=1}^{n-1} m_{i, n-j} M_{i, n-j}, \quad j \in \mathbb{N}_{1}^{n-1},
$$


where $M_{i, n-j}$ is the algebraic cofactor of $m_{i, n-j}$.

It is easy to show that

$$
\left|M_{i, n-j}\right| \leq(n-2) !=\Gamma(n-1), \quad i \in \mathbb{N}_{1}^{n-1}, \quad j \in \mathbb{N}_{1}^{n-1} .
$$

\section{Lemma 2.13 Suppose that (a)-(c) hold. Then}

(i) $L_{1}$ is a Fredholm operator of index zero.

(ii) $N_{1}: \bar{\Omega} \rightarrow Z$ is called $L_{1}$-compact for bounded set $\bar{\Omega} \sqsubseteq X$.

(iii) $x$ is a solution of $B V P(1,6)$ if and only if $L_{1} x=N_{1} x$.

Proof First, we prove that $L: D(L) \subset E \rightarrow Z$ is a Fredholm operator of index zero.

We see that

$$
\operatorname{Ker} L_{1}=\left\{c_{00} \in \mathbb{R}\right\}
$$

In fact, $x \in \operatorname{Ker} L_{1}$ if and only if

$$
\left(\begin{array}{c}
{ }^{c} D_{0^{+}}^{\alpha} x(t) \\
\Delta x\left(t_{s}\right): s \in \mathbb{N}_{1}^{m} \\
\Delta x^{\prime}\left(t_{s}\right): s \in \mathbb{N}_{1}^{m} \\
\cdots \\
\Delta x^{(n-1)}\left(t_{s}\right): s \in \mathbb{N}_{1}^{m}
\end{array}\right)=\left(\begin{array}{c}
0 \\
0: s \in \mathbb{N}_{1}^{m} \\
0: s \in \mathbb{N}_{1}^{m} \\
\cdots \\
0: s \in \mathbb{N}_{1}^{m}
\end{array}\right) .
$$

Use Lemma 2.8, we have $x \in D\left(L_{1}\right)$ and $x(t)=\sum_{w=0}^{i} \sum_{v=0}^{n-1} \frac{c_{v w}}{\Gamma(v+1)}\left(t-t_{w}\right)^{v}, t \in\left(t_{i}, t_{i+1}\right], i \in \mathbb{N}_{0}^{m}$. Then, $x(t)=c_{00}, t \in[0,1]$.

We have

$$
\operatorname{Im} L_{1}=\left\{\left(u, a_{i j}: i \in \mathbb{N}_{0}^{n-1}, j \in \mathbb{N}_{1}^{m}\right) \mid \sum_{w=1}^{m} a_{n-1 w}+\int_{0}^{1} \frac{(1-s)^{\alpha-n}}{\Gamma(\alpha-n+1)} u(s) \mathrm{d} s=0\right\} .
$$

In fact, $\left(u, a_{i j}: i \in \mathbb{N}_{0}^{n-1}, j \in \mathbb{N}_{1}^{m}\right) \in \operatorname{Im} L_{1}$ if and only if there exists $x \in D\left(L_{1}\right)$, such that

$$
\left(\begin{array}{c}
{ }^{c} D_{0^{+}}^{\alpha} x(t) \\
\Delta x\left(t_{s}\right): s \in \mathbb{N}_{1}^{m} \\
\Delta x^{\prime}\left(t_{s}\right): s \in \mathbb{N}_{1}^{m} \\
\cdots \\
\Delta x^{(n-1)}\left(t_{s}\right): s \in \mathbb{N}_{1}^{m}
\end{array}\right)=\left(\begin{array}{c}
u(t) \\
a_{1 s}: s \in \mathbb{N}_{1}^{m} \\
a_{2 s}: s \in \mathbb{N}_{1}^{m} \\
\cdots \\
a_{n-1 s}: s \in \mathbb{N}_{1}^{m}
\end{array}\right) .
$$

By Lemma 2.8, we know that there exist constants $c_{v j} \in \mathbb{R}$, such that $x^{(i)}(0)=x^{(i)}(1), i \in \mathbb{N}_{0}^{n-1}$ and

$$
x(t)=\sum_{w=0}^{s} \sum_{v=0}^{n-1} \frac{c_{v w}}{\Gamma(v+1)}\left(t-t_{w}\right)^{v}+\int_{0}^{t} \frac{(t-s)^{\alpha-1}}{\Gamma(\alpha)} u(s) \mathrm{d} s, \quad t \in\left(t_{s}, t_{s+1}\right], s \in \mathbb{N}_{0}^{m} .
$$

By Definition 2, we have for $j \in \mathbb{N}_{0}^{n-1}$ that

$x^{(j)}(t)=\sum_{w=0}^{s} \sum_{v=j}^{n-1} \frac{c_{v w}}{\Gamma(v-j+1)}\left(t-t_{w}\right)^{v-j}+\int_{0}^{t} \frac{(t-s)^{\alpha-j-1}}{\Gamma(\alpha-j)} u(s) \mathrm{d} s, \quad t \in\left(t_{s}, t_{s+1}\right], s \in \mathbb{N}_{0}^{m}, j \in \mathbb{N}_{0}^{n-1}$.

From $\Delta x^{(j)}\left(t_{s}\right)=a_{j s}$ and (2.9), we get $c_{j s}=a_{j s}\left(j \in \mathbb{N}_{0}^{n-1}, s \in \mathbb{N}_{1}^{m}\right)$. From $x^{(i)}(0)=x^{(i)}(1), i \in \mathbb{N}_{0}^{n-1}$, we have

$$
\sum_{w=1}^{m} a_{n-1 w}+\int_{0}^{1} \frac{(1-s)^{\alpha-n}}{\Gamma(\alpha-n+1)} u(s) \mathrm{d} s=0
$$


and

$$
\begin{aligned}
& \left(\begin{array}{ccccc}
\frac{1}{\Gamma(2)} & 0 & 0 & \cdots & 0 \\
\frac{1}{\Gamma(3)} & \frac{1}{\Gamma(2)} & 0 & \cdots & 0 \\
\cdots & \cdots & \cdots & \cdots & \cdots \\
\frac{1}{\Gamma(n)} & \frac{1}{\Gamma(n-1)} & \frac{1}{\Gamma(n-3)} & \cdots & \frac{1}{\Gamma(2)}
\end{array}\right)\left(\begin{array}{c}
c_{n-1} 0 \\
c_{n-2} \\
\cdots \\
c_{1} 0
\end{array}\right) \\
& =-\left(\begin{array}{c}
\sum_{w=1}^{m} \sum_{v=n-2}^{n-1} \frac{a_{v w}\left(1-t_{w}\right)^{v-(n-2)}}{\Gamma(v-(n-2)+1)}+\int_{0}^{1} \frac{(1-s)^{\alpha-(n-2)-1}}{\Gamma(\alpha-(n-2))} u(s) \mathrm{d} s \\
\sum_{w=1}^{m} \sum_{v=n-3}^{n-1} \frac{a_{v w}\left(1-t_{w}\right)^{v-(n-3)}}{\Gamma(v-(n-3)+1)}+\int_{0}^{1} \frac{(1-s)^{\alpha-(n-3)-1}}{\Gamma(\alpha-(n-3))} u(s) \mathrm{d} s \\
\cdots \\
\sum_{w=1}^{m} \sum_{v=0}^{n-1} \frac{a_{v w}\left(1-t_{w}\right)^{v}}{\Gamma(v+1)}+\int_{0}^{1} \frac{(1-s)^{\alpha-1}}{\Gamma(\alpha)} u(s) \mathrm{d} s
\end{array}\right) .
\end{aligned}
$$

Hence

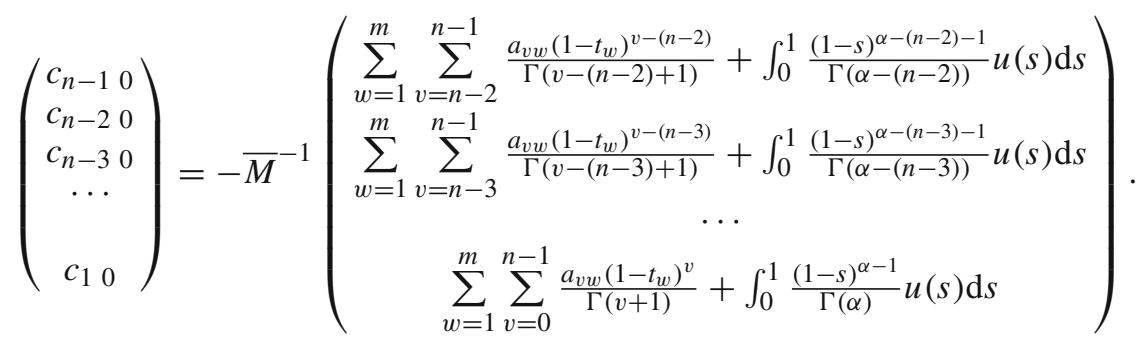

It follows for $i \in \mathbb{N}_{1}^{n-1}$ that

$$
c_{i 0}=-\sum_{j=1}^{n-1} M_{j n-i}\left(\sum_{w=1}^{m} \sum_{\sigma=n-1-j}^{n-1} \frac{\left(1-t_{w}\right)^{\sigma-(n-1-j)}}{\Gamma(\sigma-(n-1-j)+1)} a_{\sigma w}+\int_{0}^{1} \frac{(1-s)^{\alpha-(n-1-j)-1}}{\Gamma(\alpha-(n-1-j))} u(s) \mathrm{d} s\right) .
$$

We have by (2.8) that

$$
\begin{aligned}
x(t)= & c_{00}-\sum_{v=1}^{n-1} \frac{\sum_{j=1}^{n-1} M_{j n-v}\left(\sum_{w=1}^{m} \sum_{\sigma=n-1-j}^{n-1} \frac{\left(1-t_{w}\right)^{\sigma-(n-1-j)}}{\Gamma(\sigma-(n-1-j)+1)} a_{\sigma w}+\int_{0}^{1} \frac{(1-s)^{\alpha-(n-1-j)-1}}{\Gamma(\alpha-(n-1-j))} u(s) \mathrm{d} s\right)}{\Gamma(v+1)} t^{v} \\
& +\sum_{w=1}^{s} \sum_{v=0}^{n-1} \frac{a_{v w}}{\Gamma(v+1)}\left(t-t_{w}\right)^{v}+\int_{0}^{t} \frac{(t-s)^{\alpha-1}}{\Gamma(\alpha)} u(s) \mathrm{d} s, \quad t \in\left(t_{s}, t_{s+1}\right], s \in \mathbb{N}_{0}^{m} .
\end{aligned}
$$

It follows $\operatorname{dim} \operatorname{Ker} L_{1}=1$, and $\operatorname{Im} L_{1}$ is closed in $Z$. Furthermore, define projectors $P: X \rightarrow \operatorname{Ker} L_{1}$ and $Q: Z \rightarrow \operatorname{Im} L_{1}$ by

$$
\begin{aligned}
& P x(t)=x(0), x \in X \\
& Q\left(u, a_{i j}: i \in \mathbb{N}_{0}^{n-1}, j \in \mathbb{N}_{1}^{m}\right)=\left(\frac{\int_{0}^{1} \frac{(1-s)^{\alpha-n}}{\Gamma(\alpha-n+1)} u(s) \mathrm{d} s+\sum_{w=1}^{m} a_{n-1} w}{\int_{0}^{1} \frac{(1-s)^{\alpha-n}}{\Gamma(\alpha-n+1)} \mathrm{d} s}, \quad 0: i \in \mathbb{N}_{0}^{n-1}, j \in \mathbb{N}_{1}^{m}\right) .
\end{aligned}
$$

It is easy to see that $P: X \rightarrow \operatorname{Ker} L_{1}$ and $Q: Z \rightarrow \operatorname{Im} L_{1}$ are well defined and

$\operatorname{Im} P=\operatorname{Ker} L_{1}, \operatorname{Ker} Q=\operatorname{Im} L_{1}, \quad X=\operatorname{Ker} L_{1} \oplus \operatorname{Ker} P, \quad Z=\operatorname{Im} L_{1} \oplus \operatorname{Im} Q$.

Therefore, $\operatorname{dim} \operatorname{Ker} L_{1}=$ co $\operatorname{dim} \operatorname{Im} L_{1}=1<+\infty$. Then, $L_{1}: D(L) \subset X \rightarrow Z$ is a Fredholm operator of index zero.

The inverse of

$$
\left.L_{1}\right|_{D\left(L_{1}\right) \cap \operatorname{Ker} P}: D\left(L_{1}\right) \cap \operatorname{Ker} P \rightarrow \operatorname{Im} L_{1}
$$

is denoted by $K_{P}: \operatorname{Im} L_{1} \rightarrow D\left(L_{1}\right) \cap \operatorname{Ker} P$ with 


$$
\begin{aligned}
& K_{p}\left(u, a_{i j}: i \in \mathbb{N}_{0}^{n-1}, j \in \mathbb{N}_{1}^{m}\right)(t) \\
& =-\sum_{v=1}^{n-1} \frac{\sum_{j=1}^{n-1} M_{j n-v}\left(\sum_{w=1}^{m} \sum_{\sigma=n-1-j}^{n-1} \frac{\left(1-t_{w}\right)^{\sigma-(n-1-j)}}{\Gamma(\sigma-(n-1-j)+1)} a_{\sigma w}+\int_{0}^{1} \frac{(1-s)^{\alpha-(n-1-j)-1}}{\Gamma(\alpha-(n-1-j))} u(s) \mathrm{d} s\right)}{\Gamma(v+1)} t^{v} \\
& \quad+\sum_{w=1}^{s} \sum_{v=0}^{n-1} \frac{a_{v w}}{\Gamma(v+1)}\left(t-t_{w}\right)^{v}+\int_{0}^{t} \frac{(t-s)^{\alpha-1}}{\Gamma(\alpha)} u(s) \mathrm{d} s, \quad t \in\left(t_{s}, t_{s+1}\right], s \in \mathbb{N}_{0}^{m} .
\end{aligned}
$$

Second, for each non-empty open bounded subset $\Omega$ of $E$ satisfying $D\left(L_{1}\right) \cap \bar{\Omega} \neq \emptyset$, we prove that $N_{1}: \bar{\Omega} \rightarrow Z$ is $L_{1}$ compact. It suffices to prove that $Q N(\bar{\Omega})$ is bounded and $K_{p}(I-Q) N(\bar{\Omega})$ is bounded and relatively compact.

One sees that

$$
\begin{aligned}
Q N_{1} x(t) & =Q\left(\begin{array}{c}
f_{x}(t) \\
I_{0 x}\left(t_{s}\right): s \in \mathbb{N}_{1}^{m} \\
I_{1 x}\left(t_{s}\right): s \in \mathbb{N}_{1}^{m} \\
\cdots \\
I_{n-1 x}\left(t_{s}\right): s \in \mathbb{N}_{1}^{m}
\end{array}\right) \\
& =\left(\frac{\int_{0}^{1} \frac{(1-s)^{\alpha-n}}{\Gamma(\alpha-n+1)} f_{x}(s) \mathrm{d} s+\sum_{w=1}^{m} I_{n-1 x}\left(t_{w}\right)}{\int_{0}^{1} \frac{(1-s)^{\alpha-n}}{\Gamma(\alpha-n+1)} \mathrm{d} s}, 0: i \in \mathbb{N}_{0}^{n-1}, j \in \mathbb{N}_{1}^{m}\right),
\end{aligned}
$$

and by direct computation, we have

$$
\begin{aligned}
& K_{p}(I-Q) N_{1} x(t)=K_{P} N_{1} x(t)-K_{P} Q N_{1} x(t) \\
&= K_{p}\left(\begin{array}{c}
f_{x}(t) \\
I_{0 x}\left(t_{s}\right): s \in \mathbb{N}_{1}^{m} \\
I_{1 x}\left(t_{s}\right): s \in \mathbb{N}_{1}^{m} \\
\cdots \\
I_{n-1}\left(t_{s}\right): s \in \mathbb{N}_{1}^{m}
\end{array}\right)-K_{p}\left(\frac{\int_{0}^{1} \frac{(1-s)^{\alpha-n}}{\Gamma(\alpha-n+1)} f_{x}(s) \mathrm{d} s+\sum_{w=1}^{m} I_{n-1}\left(t_{w}\right)}{\int_{0}^{1} \frac{(1-s)^{\alpha-n}}{\Gamma(\alpha-n+1)} \mathrm{d} s}, 0: i \in \mathbb{N}_{0}^{n-1}, j \in \mathbb{N}_{1}^{m}\right) \\
&=-\sum_{v=1}^{n-1} \sum_{j=1}^{n-1} M_{j n-v}\left(\sum_{w=1}^{m} \sum_{\sigma=n-1-j}^{n-1} \frac{\left(1-t_{w}\right)^{\sigma-(n-1-j)}}{\Gamma(\sigma-(n-1-j)+1)} I_{\sigma x}\left(t_{w}\right)+\int_{0}^{1} \frac{(1-s)^{\alpha-(n-1-j)-1}}{\Gamma(\alpha-(n-1-j))} f_{x}(s) \mathrm{d} s\right) \frac{t^{v}}{\Gamma(v+1)} \\
&+\Gamma(\alpha-n+2)\left(\int_{0}^{1} \frac{(1-s)^{\alpha-n}}{\Gamma(\alpha-n+1)} f_{x}(s) \mathrm{d} s+\sum_{w=1}^{m} I_{n-1}\left(t_{w}\right)\right) \sum_{v=1}^{n-1} \sum_{j=1}^{n-1} \frac{M_{j}}{\Gamma(\alpha-v-(n-1-j)+1)} \frac{M^{v}}{\Gamma(v+1)} \\
&-\Gamma(\alpha-n+2)\left(\int_{0}^{1} \frac{(1-s)^{\alpha-n}}{\Gamma(\alpha-n+1)} f_{x}(s) \mathrm{d} s+\sum_{w=1}^{m} I_{n-1}\left(t_{w}\right)\right) \frac{t^{\alpha}}{\Gamma(\alpha+1)} \\
&+\sum_{w=1}^{s} \sum_{v=0}^{n-1} \frac{I_{v x}\left(t_{x}\right)}{\Gamma(v+1)}\left(t-t_{w}\right)^{v}+\int_{0}^{t} \frac{(t-s)^{\alpha-1}}{\Gamma(\alpha)} f_{x}(s) \mathrm{d} s, \quad t \in\left(t_{s}, t_{s+1}\right], s \in \mathbb{N}_{0}^{m} .
\end{aligned}
$$

By Lemma 2.12, we can prove that $Q N(\bar{\Omega})$ is bounded and $K_{p}(I-Q) N(\bar{\Omega})$ is bounded and relatively compact. Hence, $N_{1}: \bar{\Omega} \rightarrow Z$ is called $L_{1}$-compact for bounded set $\bar{\Omega} \sqsubseteq X$.

Third, it is easy to see that $x$ is a solution of $\operatorname{BVP}(1,6)$ if and only if $L_{1} x=N_{1} x$. The proof is complete.

\section{Main results}

In this section, we are ready to present the main theorems. We need the following assumptions:

A function $\prod:[0, \infty) \times[0, \infty) \rightarrow[0, \infty)$ is called a bi-non-increasing function if both $x \rightarrow \prod(x, y)$ and $y \rightarrow \prod(x, y)$ are non-increasing. We need the following assumptions: 
(H1) there exist non-negative bi-non-decreasing functions $\prod_{f}, \prod_{j}:[0, \infty) \times[0, \infty) \rightarrow[0, \infty)$, such that

$$
\begin{aligned}
& |f(t, x, y)| \leq \prod_{f}(|x|,|y|) t^{p}(1-t)^{q}, \quad t \in\left(t_{s}, t_{s+1}\right], s \in \mathbb{N}_{0}^{m}, \\
& \left|I_{j}\left(t_{s}, x, y\right)\right| \leq \prod_{I}(|x|,|y|), \quad s \in \mathbb{N}_{1}^{m}, j \in \mathbb{N}_{0}^{n-1} .
\end{aligned}
$$

(H2) there exists a constant $M>0$, such that for $x \in X$ with $|x(t)|>M$ for all $t \in(0,1]$ implies that

$$
\int_{0}^{1} \frac{(1-t)^{\alpha-n}}{\Gamma(\alpha-n+1)} f\left(t, x(t),{ }^{c} D_{0^{+}}^{\beta} x(t)\right) \mathrm{d} t+\sum_{w=1}^{m} I_{n-1}\left(t_{w}, x\left(t_{w}\right),{ }^{c} D_{0^{+}}^{\beta} x\left(t_{s}\right)\right) \neq 0 .
$$

(H3) there exists a constant $M_{0}>0$, such that

$$
c\left[\int_{0}^{1} \frac{(1-t)^{\alpha-n}}{\Gamma(\alpha-n+1)} f(t, c, 0) \mathrm{d} t+\sum_{w=1}^{m} I_{n-1}\left(t_{w}, c, 0\right]>0\right.
$$

holds for all $|c|>M_{0}$ or

$$
c\left[\int_{0}^{1} \frac{(1-t)^{\alpha-n}}{\Gamma(\alpha-n+1)} f(t, c, 0) \mathrm{d} t+\sum_{w=1}^{m} I_{n-1}\left(t_{w}, c, 0\right]<0 .\right.
$$

holds for all $|c|>M_{0}$.

Theorem 3.1 Suppose that (a)-(c) and (H1)-(H3) hold. Then, BVP (1.7) has at least one solution if

$$
\lim _{r \rightarrow \infty} \frac{r}{M+M_{1} \prod_{I}(r, r)+M_{2} \prod_{f}(r, r)}>1,
$$

where

$$
\begin{aligned}
M_{1}= & 2 \sum_{v=1}^{n-1} \sum_{j=1}^{n-1} \frac{\Gamma(n-1)}{\Gamma(v+1)} \sum_{w=1}^{m} \sum_{\sigma=n-1-j}^{n-1} \frac{\left(1-t_{w}\right)^{\sigma-(n-1-j)}}{\Gamma(\sigma-(n-1-j)+1)} \\
& +\sum_{v=1}^{n-1} \sum_{j=1}^{n-1} \frac{\Gamma(n-1)}{\Gamma(v-\beta+1)} \sum_{w=1}^{m} \sum_{\sigma=n-1-j}^{n-1} \frac{\left(1-t_{w}\right)^{\sigma-(n-1-j)}}{\Gamma(\sigma-(n-1-j)+1)} \\
& +2 \sum_{v=0}^{n-1} \frac{m}{\Gamma(v+1)}+\sum_{v=0}^{n-1} \frac{m}{\Gamma(v-\beta+1)}, \\
M_{2}= & \sum_{v=1}^{n-1} \sum_{j=1}^{n-1} \frac{\Gamma(n-1)}{\Gamma(v+1)} \frac{\mathbf{B}(\alpha+q-(n-1-j), p+1)}{\Gamma(\alpha-(n-1-j))}+2 \frac{\mathbf{B}(\alpha+q, p+1)}{\Gamma(\alpha)} \\
& +\sum_{v=1}^{n-1} \sum_{j=1}^{n-1} \frac{\Gamma(n-1)}{\Gamma(v-\beta+1)} \frac{\mathbf{B}(\alpha+q-(n-1-j), p+1)}{\Gamma(\alpha-(n-1-j))}+\frac{\mathbf{B}(\alpha+q-\beta, p+1)}{\Gamma(\alpha-\beta)} .
\end{aligned}
$$

Proof Let $X, Z, L_{1}$, and $N_{1}$ be defined in Sect. 2. By (a)-(c) and (H1)-(H3), from Lemma 2.7, $L_{1}$ be a Fredholm operator of index zero and $N_{1}$ be $L$ compact on each closed non-empty set $\Omega$ centered at zero. We seek fixed point of the operator equation $L_{x}=N_{1} x$. To apply Lemma 2.7, we should define an open bounded subset $\Omega$ of $X$ centered at zero, such that (i), (ii), and (iii) in Lemma 2.7 hold. To obtain $\Omega$, we do three steps. The proof of this theorem is divided into four steps.

Step 1 Let $\Omega_{1}=\left\{x \in X \cap D\left(L_{1}\right) \backslash \operatorname{Ker} L_{1}, L_{1} x=\lambda N_{1} x\right.$ for some $\left.\lambda \in(0,1)\right\}$. We prove that $\Omega_{1}$ is bounded. 
In fact, for $x \in \Omega_{1}$, we have $L_{1} x=\lambda N_{1} x$ and $N_{1} x \in \operatorname{Im} L_{1}$. Then

$$
\begin{cases}{ }^{c} D_{0^{+}}^{\alpha} x(t)=\lambda f\left(t, x(t),{ }^{c} D_{0^{+}}^{\beta} x(t)\right), & t \in\left(t_{s}, t_{s+1}\right], s \in \mathbb{N}_{0}^{m}, \\ x^{(j)}(0)=x^{(j)}(1), & j \in \mathbb{N}_{0}^{n-1}, \\ \Delta x^{(j)}\left(t_{s}\right)=\lambda_{u} I_{j}\left(t_{s}, x\left(t_{s}\right),{ }^{c} D_{0^{+}}^{\beta} x\left(t_{s}\right)\right), & j \in \mathbb{N}_{0}^{n-1}, s \in \mathbb{N}_{1}^{m} .\end{cases}
$$

So

$$
\left.\int_{0}^{1} \frac{(1-s)^{\alpha-n}}{\Gamma(\alpha-n+1)} f\left(s, x(s),{ }^{c} D_{0^{+}}^{\beta} x(s)\right) \mathrm{d} s+\sum_{w=1}^{m} I_{n-1}\left(t_{w}, x\left(t_{w}\right),{ }^{c} D_{0^{+}}^{\beta} x\left(t_{w}\right)\right)\right)=0 .
$$

It follows from $(\mathrm{H} 1)$ that

$$
\begin{aligned}
\left|f\left(t, x(t),{ }^{c} D_{0^{+}}^{\beta} x(t)\right)\right| & \leq \prod_{f}\left(|x(t)|,\left|{ }^{c} D_{0^{+}}^{\beta} x(t)\right|\right) t^{p}(1-t)^{q} \\
& \leq \prod_{f}(\|x\|,\|x\|) t^{p}(1-t)^{q}, \quad t \in\left(t_{s}, t_{s+1}\right], s \in \mathbb{N}_{0}^{m}, \\
\left|I_{j}\left(t_{s}, x\left(t_{s}\right),{ }^{c} D_{0^{+}}^{\beta} x\left(t_{s}\right)\right)\right| & \leq \prod_{I}(\|x\|,\|x\|), \quad s \in \mathbb{N}_{1}^{m}, j \in \mathbb{N}_{0}^{n-1} .
\end{aligned}
$$

It follows from (H2) that there exists $\bar{t} \in\left(t_{s}, t_{s+1}\right]$ (for some $\left.s \in \mathbb{N}_{0}^{m}\right)$, such that $|x(\bar{t})| \leq M$. By similar method used in (2.11), we have

$$
\begin{aligned}
x(t)= & x(0)-\lambda \sum_{v=1}^{n-1} \sum_{j=1}^{n-1} M_{j n-v} \sum_{w=1}^{m} \sum_{\sigma=n-1-j}^{n-1} \frac{\left(1-t_{w}\right)^{\sigma-(n-1-j)}}{\Gamma(\sigma-(n-1-j)+1)} I_{\sigma x}\left(t_{w}\right) \frac{t^{v}}{\Gamma(v+1)} \\
& +\lambda \sum_{v=1}^{n-1} \sum_{j=1}^{n-1} M_{j n-v} \int_{0}^{1} \frac{(1-s)^{\alpha-(n-1-j)-1}}{\Gamma(\alpha-(n-1-j))} f_{x}(s) \mathrm{d} s \frac{t^{v}}{\Gamma(v+1)} \\
& +\lambda \sum_{w=1}^{s} \sum_{v=0}^{n-1} \frac{I_{v x}\left(t_{w}\right)}{\Gamma(v+1)}\left(t-t_{w}\right)^{v}+\lambda \int_{0}^{t} \frac{(t-s)^{\alpha-1}}{\Gamma(\alpha)} f_{x}(s) \mathrm{d} s, \quad t \in\left(t_{s}, t_{s+1}\right], s \in \mathbb{N}_{0}^{m} .
\end{aligned}
$$

Then

$$
\begin{aligned}
|x(0)| \leq & |x(\bar{t})|+\lambda \sum_{v=1}^{n-1} \sum_{j=1}^{n-1}\left|M_{j} n-v\right| \sum_{w=1}^{m} \sum_{\sigma=n-1-j}^{n-1} \frac{\left(1-t_{w}\right)^{\sigma-(n-1-j)}}{\Gamma(\sigma-(n-1-j)+1)}\left|I_{\sigma x}\left(t_{w}\right)\right| \frac{\bar{t}^{v}}{\Gamma(v+1)} \\
& +\lambda \sum_{v=1}^{n-1} \sum_{j=1}^{n-1}\left|M_{j n-v}\right| \int_{0}^{1} \frac{(1-s)^{\alpha-(n-1-j)-1}}{\Gamma(\alpha-(n-1-j))}\left|f_{x}(s)\right| \mathrm{d} s \frac{t^{v}}{\Gamma(v+1)} \\
& +\lambda \sum_{w=1}^{s} \sum_{v=0}^{n-1} \frac{\left|I_{v x}\left(t_{w}\right)\right|}{\Gamma(v+1)}\left(\bar{t}-t_{w}\right)^{v}+\lambda \int_{0}^{\bar{t}} \frac{(\bar{t}-s)^{\alpha-1}}{\Gamma(\alpha)}\left|f_{x}(s)\right| \mathrm{d} s \\
\leq & M+\sum_{v=1}^{n-1} \sum_{j=1}^{n-1} \Gamma(n-1) \sum_{w=1}^{m} \sum_{\sigma=n-1-j}^{n-1} \frac{\left(1-t_{w}\right)^{\sigma-(n-1-j)}}{\Gamma(\sigma-(n-1-j)+1)} \prod_{I}(\|x\|,\|x\|) \frac{1}{\Gamma(v+1)} \\
& +\sum_{v=1}^{n-1} \sum_{j=1}^{n-1} \Gamma(n-1) \int_{0}^{1} \frac{(1-s)^{\alpha-(n-1-j)-1}}{\Gamma(\alpha-(n-1-j))} \prod_{f}(\|x\|,\|x\|) s^{p}(1-s)^{q} \mathrm{~d} s \frac{1}{\Gamma(v+1)}
\end{aligned}
$$




$$
\begin{aligned}
& +\sum_{w=1}^{s} \sum_{v=0}^{n-1} \frac{\prod_{I}(\|x\|,\|x\|)}{\Gamma(v+1)}+\int_{0}^{\bar{t}} \frac{(\bar{t}-s)^{\alpha-1}}{\Gamma(\alpha)} \prod_{f}(\|x\|,\|x\|) s^{p}(1-s)^{q} \mathrm{~d} s \\
\leq & M+\sum_{v=1}^{n-1} \sum_{j=1}^{n-1} \frac{\Gamma(n-1)}{\Gamma(v+1)} \sum_{w=1}^{m} \sum_{\sigma=n-1-j}^{n-1} \frac{\left(1-t_{w}\right)^{\sigma-(n-1-j)}}{\Gamma(\sigma-(n-1-j)+1)} \prod_{I}(\|x\|,\|x\|) \\
+ & \sum_{v=1}^{n-1} \sum_{j=1}^{n-1} \frac{\Gamma(n-1)}{\Gamma(v+1)} \frac{\mathbf{B}(\alpha+q-(n-1-j), p+1)}{\Gamma(\alpha-(n-1-j))} \prod_{f}(\|x\|,\|x\|) \\
& +\sum_{v=0}^{n-1} \frac{m}{\Gamma(v+1)} \prod_{I}(\|x\|,\|x\|)+\frac{\mathbf{B}(\alpha+q, p+1)}{\Gamma(\alpha)} \prod_{f}(\|x\|,\|x\|) .
\end{aligned}
$$

From (3.3), we have similarly that

$$
\begin{aligned}
|x(t)| \leq & |x(0)|+\sum_{v=1}^{n-1} \sum_{j=1}^{n-1}\left|M_{j} n-v\right| \sum_{w=1}^{m} \sum_{\sigma=n-1-j}^{n-1} \frac{\left(1-t_{w}\right)^{\sigma-(n-1-j)}}{\Gamma(\sigma-(n-1-j)+1)}\left|I_{\sigma x}\left(t_{w}\right)\right| \frac{t^{v}}{\Gamma(v+1)} \\
& +\sum_{v=1}^{n-1} \sum_{j=1}^{n-1}\left|M_{j} n-v\right| \int_{0}^{1} \frac{(1-s)^{\alpha-(n-1-j)-1}}{\Gamma(\alpha-(n-1-j))}\left|f_{x}(s)\right| \mathrm{d} s \frac{t^{v}}{\Gamma(v+1)} \\
& +\sum_{w=1}^{s} \sum_{v=0}^{n-1} \frac{\left|I_{v x}\left(t_{w}\right)\right|}{\Gamma(v+1)}\left(t-t_{w}\right)^{v}+\int_{0}^{t} \frac{(t-s)^{\alpha-1}}{\Gamma(\alpha)}\left|f_{x}(s)\right| \mathrm{d} s \\
\leq & M+2 \sum_{v=1}^{n-1} \sum_{j=1}^{n-1} \frac{\Gamma(n-1)}{\Gamma(v+1)} \sum_{w=1}^{m} \sum_{\sigma=n-1-j}^{n-1} \frac{\left(1-t_{w}\right)^{\sigma-(n-1-j)}}{\Gamma(\sigma-(n-1-j)+1)} \prod_{I}(\|x\|,\|x\|) \\
& +2 \sum_{v=1}^{n-1} \sum_{j=1}^{n-1} \frac{\Gamma(n-1)}{\Gamma(v+1)} \frac{\mathbf{B}(\alpha+q-(n-1-j), p+1)}{\Gamma(\alpha-(n-1-j))} \prod_{f}(\|x\|,\|x\|) \\
& +2 \sum_{v=0}^{n-1} \frac{m(v+1)}{\Gamma(x)} \prod_{I}(\|x\|,\|x\|)+2 \frac{\mathbf{B}(\alpha+q, p+1)}{\Gamma(\alpha)} \prod_{f}(\|x\|,\|x\|) .
\end{aligned}
$$

It follows from (3.3) and Definition 2.2 that

$$
\begin{aligned}
{ }^{c} D_{0^{+}}^{\beta} x(t)= & -\lambda \sum_{v=1}^{n-1} \sum_{j=1}^{n-1} M_{j} n-v \sum_{w=1}^{m} \sum_{\sigma=n-1-j}^{n-1} \frac{\left(1-t_{w}\right)^{\sigma-(n-1-j)}}{\Gamma(\sigma-(n-1-j)+1)} I_{\sigma x}\left(t_{w}\right) \frac{t^{v-\beta}}{\Gamma(v-\beta+1)} \\
& +\lambda \sum_{v=1}^{n-1} \sum_{j=1}^{n-1} M_{j} n-v \int_{0}^{1} \frac{(1-s)^{\alpha-(n-1-j)-1}}{\Gamma(\alpha-(n-1-j))} f_{x}(s) \mathrm{d} s \frac{t^{v-\beta}}{\Gamma(v-\beta+1)} \\
& +\lambda \sum_{w=1}^{s} \sum_{v=0}^{n-1} \frac{I_{v x}\left(t_{w}\right)}{\Gamma(v-\beta+1)}\left(t-t_{w}\right)^{v-\beta}+\lambda \int_{0}^{t} \frac{(t-s)^{\alpha-\beta-1}}{\Gamma(\alpha-\beta)} f_{x}(s) \mathrm{d} s, \quad t \in\left(t_{s}, t_{s+1}\right], s \in \mathbb{N}_{0}^{m} .
\end{aligned}
$$


So

$$
\begin{aligned}
& \left|{ }^{c} D_{0^{+}}^{\beta} x(t)\right| \leq \sum_{v=1}^{n-1} \sum_{j=1}^{n-1}\left|M_{j n-v}\right| \sum_{w=1}^{m} \sum_{\sigma=n-1-j}^{n-1} \frac{\left(1-t_{w}\right)^{\sigma-(n-1-j)}}{\Gamma(\sigma-(n-1-j)+1)}\left|I_{\sigma x}\left(t_{w}\right)\right| \frac{1}{\Gamma(v-\beta+1)} \\
& +\sum_{v=1}^{n-1} \sum_{j=1}^{n-1}\left|M_{j n-v}\right| \int_{0}^{1} \frac{(1-s)^{\alpha-(n-1-j)-1}}{\Gamma(\alpha-(n-1-j))}\left|f_{x}(s)\right| \mathrm{d} s \frac{1}{\Gamma(v-\beta+1)} \\
& +\sum_{w=1}^{s} \sum_{v=0}^{n-1} \frac{\left|I_{v x}\left(t_{w}\right)\right|}{\Gamma(v-\beta+1)}+\int_{0}^{t} \frac{(t-s)^{\alpha-\beta-1}}{\Gamma(\alpha-\beta)}\left|f_{x}(s)\right| \mathrm{d} s \\
& \leq \sum_{v=1}^{n-1} \sum_{j=1}^{n-1} \frac{\Gamma(n-1)}{\Gamma(v-\beta+1)} \sum_{w=1}^{m} \sum_{\sigma=n-1-j}^{n-1} \frac{\left(1-t_{w}\right)^{\sigma-(n-1-j)}}{\Gamma(\sigma-(n-1-j)+1)} \prod_{I}(\|x\|,\|x\|) \\
& +\sum_{v=1}^{n-1} \sum_{j=1}^{n-1}\left|M_{j n-v}\right| \int_{0}^{1} \frac{(1-s)^{\alpha-(n-1-j)-1}}{\Gamma(\alpha-(n-1-j))} \prod_{f}(\|x\|,\|x\|) s^{p}(1-s)^{q} \mathrm{~d} s \frac{1}{\Gamma(v-\beta+1)} \\
& +\sum_{w=1}^{s} \sum_{v=0}^{n-1} \frac{\prod_{I}(\|x\|,\|x\|)}{\Gamma(v-\beta+1)}+\int_{0}^{t} \frac{(t-s)^{\alpha-\beta-1}}{\Gamma(\alpha-\beta)} \prod_{f}(\|x\|,\|x\|) s^{p}(1-s)^{q} \mathrm{~d} s \\
& \leq \sum_{v=1}^{n-1} \sum_{j=1}^{n-1} \frac{\Gamma(n-1)}{\Gamma(v-\beta+1)} \sum_{w=1}^{m} \sum_{\sigma=n-1-j}^{n-1} \frac{\left(1-t_{w}\right)^{\sigma-(n-1-j)}}{\Gamma(\sigma-(n-1-j)+1)} \prod_{I}(\|x\|,\|x\|) \\
& +\sum_{v=1}^{n-1} \sum_{j=1}^{n-1} \frac{\Gamma(n-1)}{\Gamma(v-\beta+1)} \frac{\mathbf{B}(\alpha+q-(n-1-j), p+1)}{\Gamma(\alpha-(n-1-j))} \prod_{f}(\|x\|,\|x\|) \\
& +\sum_{v=0}^{n-1} \frac{m}{\Gamma(v-\beta+1)} \prod_{I}(\|x\|,\|x\|)+\frac{\mathbf{B}(\alpha+q-\beta, p+1)}{\Gamma(\alpha-\beta)} \prod_{f}(\|x\|,\|x\|) .
\end{aligned}
$$

Hence

$$
\begin{aligned}
\|x\|= & \max \left\{\sup _{t \in(0,1]}|x(t)|, \sup _{t \in(0,1]}\left|{ }^{c} D_{0^{+}}^{\beta} x(t)\right|\right\} \\
\leq & \sup _{t \in(0,1]}|x(t)|+\sup _{t \in(0,1]}\left|{ }^{c} D_{0^{+}}^{\beta} x(t)\right| \\
\leq & M+2 \sum_{v=1}^{n-1} \sum_{j=1}^{n-1} \frac{\Gamma(n-1)}{\Gamma(v+1)} \sum_{w=1}^{m} \sum_{\sigma=n-1-j}^{n-1} \frac{\left(1-t_{w}\right)^{\sigma-(n-1-j)}}{\Gamma(\sigma-(n-1-j)+1)} \prod_{I}(\|x\|,\|x\|) \\
& +2 \sum_{v=1}^{n-1} \sum_{j=1}^{n-1} \frac{\Gamma(n-1)}{\Gamma(v+1)} \frac{\mathbf{B}(\alpha+q-(n-1-j), p+1)}{\Gamma(\alpha-(n-1-j))} \prod_{f}(\|x\|,\|x\|) \\
& +2 \sum_{v=0}^{n-1} \frac{m}{\Gamma(v+1)} \prod_{I}(\|x\|,\|x\|)+2 \frac{\mathbf{B}(\alpha+q, p+1)}{\Gamma(\alpha)} \prod_{f}(\|x\|,\|x\|) \\
& +\sum_{v=1}^{n-1} \sum_{j=1}^{n-1} \frac{\Gamma(n-1)}{\Gamma(v-\beta+1)} \sum_{w=1}^{m} \sum_{\sigma=n-1-j}^{n-1} \frac{\left(1-t_{w}\right)^{\sigma-(n-1-j)}}{\Gamma(\sigma-(n-1-j)+1)} \prod_{I}(\|x\|,\|x\|)
\end{aligned}
$$




$$
\begin{aligned}
&+ \sum_{v=1}^{n-1} \sum_{j=1}^{n-1} \frac{\Gamma(n-1)}{\Gamma(v-\beta+1)} \frac{\mathbf{B}(\alpha+q-(n-1-j), p+1)}{\Gamma(\alpha-(n-1-j))} \prod_{f}(\|x\|,\|x\|) \\
&+ \sum_{v=0}^{n-1} \frac{m}{\Gamma(v-\beta+1)} \prod_{I}(\|x\|,\|x\|)+\frac{\mathbf{B}(\alpha+q-\beta, p+1)}{\Gamma(\alpha-\beta)} \prod_{f}(\|x\|,\|x\|) \\
&=+\left(2 \sum_{v=1}^{n-1} \sum_{j=1}^{n-1} \frac{\Gamma(n-1)}{\Gamma(v+1)} \sum_{w=1}^{m} \sum_{\sigma=n-1-j}^{n-1} \frac{\left(1-t_{w}\right)^{\sigma-(n-1-j)}}{\Gamma(\sigma-(n-1-j)+1)}\right. \\
&+ \sum_{v=1}^{n-1} \sum_{j=1}^{n-1} \frac{\Gamma(n-1)}{\Gamma(v-\beta+1)} \sum_{w=1}^{m} \sum_{\sigma=n-1-j}^{n-1} \frac{\left(1-t_{w}\right)^{\sigma-(n-1-j)}}{\Gamma(\sigma-(n-1-j)+1)} \\
&+\left.2 \sum_{v=0}^{n-1} \frac{m}{\Gamma(v+1)}+\sum_{v=0}^{n-1} \frac{m}{\Gamma(v-\beta+1)}\right) \prod_{I}(\|x\|,\|x\|) \\
&+\left(2 \sum_{v=1}^{n-1} \sum_{j=1}^{n-1} \frac{\Gamma(n-1)}{\Gamma(v+1)} \frac{\mathbf{B}(\alpha+q-(n-1-j), p+1)}{\Gamma(\alpha-(n-1-j))}+2 \frac{\mathbf{B}(\alpha+q, p+1)}{\Gamma(\alpha)}\right. \\
&\left.+\sum_{v=1}^{n-1} \sum_{j=1}^{n-1} \frac{\Gamma(n-1)}{\Gamma(v-\beta+1)} \frac{\mathbf{B}(\alpha+q-(n-1-j), p+1)}{\Gamma(\alpha-(n-1-j))}+\frac{\mathbf{B}(\alpha+q-\beta, p+1)}{\Gamma(\alpha-\beta)}\right) \prod(\|x\|,\|x\|) \\
& f
\end{aligned}
$$

It follows that

$$
\|x\| \leq M+M_{1} \prod_{I}(\|x\|,\|x\|)+M_{2} \prod_{f}(\|x\|,\|x\|) .
$$

Since $\lim _{r \rightarrow \infty} \frac{r}{M+M_{1} \prod_{I}(r, r)+M_{2} \prod_{f}(r, r)}>1$, we get from (3.4) that there exists a constant $M_{4}>0$ independent of $\lambda$, such that $\|x\| \leq M_{4}$. It follows that $\Omega_{1}$ is bounded.

Step 2 Let $\Omega_{2}=\left\{c \in \operatorname{Ker} L_{1}: N_{1}(c) \in \operatorname{Im} L_{1}\right\}$. We prove that $\Omega_{2}$ is bounded.

For $c \in \Omega_{2}$, we have

$$
N_{1}(c)=\left(\begin{array}{c}
f(t, c, 0) \\
I_{0}\left(t_{s}, c, 0\right): s \in \mathbb{N}_{1}^{m} \\
I_{1}\left(t_{s}, c, 0\right): s \in \mathbb{N}_{1}^{m} \\
\cdots \\
I_{n-1}\left(t_{s} . c,\right): s \in \mathbb{N}_{1}^{m}
\end{array}\right)
$$

So, $\int_{0}^{1} \frac{(1-s)^{\alpha-n}}{\Gamma(\alpha-n+1)} f(s, c, 0) \mathrm{d} s+\sum_{w=1}^{m} I_{n-1}\left(t_{s}, c, 0\right)=0$. From (H3), we get that $|c| \leq M_{0}$. This shows that $\Omega_{2}$ is bounded.

Step 3 If the first inequality in (H3) holds, we prove that $\Omega_{3}=\left\{c \in \operatorname{Ker} L_{1}: \lambda \wedge(c)+(1-\lambda) Q N_{1}(c)=\right.$ $0, \lambda \in[0,1]\}$ is bounded, where $\wedge: \operatorname{Ker} L \rightarrow Z / \operatorname{Im} L_{1}$ is the isomorphism given by $\wedge(c)=(c, 0: i \in$ $\mathbb{N}_{0}^{n-1}, j \in \mathbb{N}_{1}^{m}$ ).

For $c \in \operatorname{Ker} L_{1}$, one sees that

$$
\begin{aligned}
-\lambda \wedge(c)= & -\lambda\left(c, 0: i \in \mathbb{N}_{0}^{n-1}, j \in \mathbb{N}_{1}^{m}\right) \\
& =(1-\lambda)\left(\frac{\int_{0}^{1} \frac{(1-s)^{\alpha-n}}{\Gamma(\alpha-n+1)} f(s, c, 0) \mathrm{d} s+\sum_{w=1}^{m} I_{n-1}\left(t_{w}, c, 0\right)}{\int_{0}^{1} \frac{(1-s)^{\alpha-n}}{\Gamma(\alpha-n+1)} \mathrm{d} s}, 0: i \in \mathbb{N}_{0}^{n-1}, j \in \mathbb{N}_{1}^{m}\right) .
\end{aligned}
$$


Then

$$
-\lambda c^{2}=(1-\lambda) \frac{c\left[\int_{0}^{1} \frac{(1-s)^{\alpha-n}}{\Gamma(\alpha-n+1)} f(s, c, 0) \mathrm{d} s+\sum_{w=1}^{m} I_{n-1}\left(t_{w}, c, 0\right)\right]}{\int_{0}^{1} \frac{(1-s)^{\alpha-n}}{\Gamma(\alpha-n+1)} \mathrm{d} s} .
$$

If $\lambda=1$, then $c=0$. If $\lambda \in[0,1)$, and $|c|>M_{0}$, we get

$$
0 \geq-\lambda c^{2}=(1-\lambda)(1-\lambda) \frac{c\left[\int_{0}^{1} \frac{(1-s)^{\alpha-n}}{\Gamma(\alpha-n+1)} f(s, c, 0) \mathrm{d} s+\sum_{w=1}^{m} I_{n-1}\left(t_{w}, c, 0\right)\right]}{\int_{0}^{1} \frac{(1-s)^{\alpha-n}}{\Gamma(\alpha-n+1)} \mathrm{d} s}>0,
$$

a contradiction. Then, $|c| \leq M_{0}$. Then, $\Omega_{3}$ is bounded.

If the second inequality in (H3) holds for all $|c|>M_{0}$, let

$$
\Omega_{3}=\left\{c \in \operatorname{Ker} L_{1}: \lambda \wedge(c)-(1-\lambda) Q N_{1}(c)=0, \lambda \in[0,1]\right\},
$$

We can prove that $\Omega_{3}$ is bounded too.

Step 4 We shall show that all conditions of Lemma 2.7 are satisfied.

Set $\Omega$ be a open bounded subset of $X$ centered at zero, such that $\Omega \supset \bigcup_{i=1}^{3} \overline{\Omega_{i}}$. By Lemma 2.13, $L_{1}$ is a Fredholm operator of index zero and $N_{1}$ is $L_{1}$ compact on $\bar{\Omega}$. By the definition of $\Omega$, we have

(a) $L_{1}(x) \neq \lambda N(x)$ for $x \in\left(D\left(L_{1}\right) \backslash \operatorname{Ker} L_{1}\right) \cap \partial \Omega$ and $\lambda \in(0,1)$;

(b) $N_{1}(x) \notin \operatorname{Im} L_{1}$ for $x \in \operatorname{Ker} L_{1} \cap \partial \Omega$.

(c) $\operatorname{deg}\left(\left.Q N_{1}\right|_{\operatorname{Ker} L_{1}}, \Omega \cap \operatorname{Ker} L_{1}, 0\right) \neq 0$. In fact, let $H(x, \lambda)= \pm \lambda \wedge(x)+(1-\lambda) Q N_{1}(x)$.

According to the definition of $\Omega$, we know $H(x, \lambda) \neq 0$ for $x \in \partial \Omega \cap \operatorname{Ker} L_{1}$, thus by homotopy property of degree,

$$
\begin{gathered}
\operatorname{deg}\left(\left.Q N_{1}\right|_{\operatorname{Ker} L_{1}}, \Omega \cap \operatorname{Ker} L_{1}, 0\right)=\operatorname{deg}\left(H(\cdot, 0), \Omega \cap \operatorname{Ker} L_{1}, 0\right) \\
=\operatorname{deg}\left(H(\cdot, 1), \Omega \cap \operatorname{Ker} L_{1}, 0\right)=\operatorname{deg}\left(\wedge, \Omega \cap \operatorname{Ker} L_{1}, 0\right) \neq 0 .
\end{gathered}
$$

Thus, by Lemma 2.7, $L_{1} x=N_{1} x$ has at least one solution in $D\left(L_{1}\right) \cap \bar{\Omega}$. Then, $x$ is a solution of BVP (1.7). The proof is complete.

Remark 3.2 Readers should try to study the solvability of the following BVP and

$$
\begin{cases}{ }^{c} D_{0^{+}}^{\gamma} u(t)=g\left(t, u(t),{ }^{c} D_{0^{+}}^{\delta} u(t)\right), & t \in\left(t_{s}, t_{s+1}\right], s \in \mathbb{N}_{0}^{m}, \\ u^{(2 i+1)}(0)=-u^{(2 i+1)}(1)=0, & i \in \mathbb{N}_{0}^{n-1}, \\ \Delta u^{(j)}\left(t_{s}\right)=J_{j}\left(t_{s}, u\left(t_{s}\right),{ }^{c} D_{0^{+}}^{\beta} u\left(t_{s}\right)\right), & j \in \mathbb{N}_{0}^{n-1}, s \in \mathbb{N}_{1}^{m},\end{cases}
$$

where $2 n-1 \leq \gamma<2 n$, and $\delta \in(0,2 n-\gamma), g$ is a Carathéodory function, and $J_{j}\left(j \in \mathbb{N}_{0}^{2 n-1}\right)$ are discrete Carathéodory functions. This problem may be called Neumann-type boundary value problem for higher order fractional differential equations.

\section{Examples}

To illustrate the usefulness of our main result, we present two examples that Theorem 3.1 can readily apply. The first one is a generalization of periodic boundary value problem for impulsive eighth order differential equation. The studies on boundary value problems for eighth order differential equations have been made in $[5,13]$. The second one corrects a mistake occurred in a known published paper. 
Example 4.1 Consider the following impulsive boundary value problem:

$$
\left\{\begin{array}{l}
{ }^{c} D_{0^{+}}^{\frac{38}{5}} u(t)=t^{-\frac{1}{5}}(1-t)^{-\frac{1}{5}}\left[c_{0}+b_{0} u(t)+a_{0} \arctan \left({ }^{c} D_{0^{+}}^{\frac{1}{5}} u(t)\right)\right], t \in\left(t_{s}, t_{s+1}\right], s \in \mathbb{N}_{0}^{1}, \\
u^{(i)}(0)=u^{(i)}(1), \quad i \in \mathbb{N}_{0}^{7}, \quad \Delta u^{(j)}(1 / 2)=B_{j 0} u(1 / 2),
\end{array}\right.
$$

where $c_{0}, b_{0}, a_{0}, C_{0}, B_{j 0}\left(j \in \mathbb{N}_{1}^{n-1}\right)$, and $A_{0}$ are non-negative constants with $b_{0}+B_{70}>0, B_{00}>-1$.

Corresponding to system (1.7) we have

(a) $\alpha=\frac{38}{5}, \beta=\frac{1}{5}, p=-\frac{1}{5}, q=-\frac{1}{5}$,

(b) $0=t_{0}<t_{1}=\frac{1}{2}<t_{2}=1$ with $m=1$ and $n=8$, and

(c) $f$ and $I_{j}$ are as follows:

$$
f(t, x, y)=t^{-\frac{1}{5}}(1-t)^{-\frac{1}{5}}\left[c_{0}+b_{0} x+a_{0} \arctan y\right], \quad I_{j}\left(t_{s}, x, y\right)=B_{j} 0 x, j \in \mathbb{N}_{0}^{7} .
$$

Choose $\prod_{f}(x, y)=c_{0}+b_{0} x+a_{0} \arctan y$ and $\prod_{I}(x, y)=\max \left\{\left|B_{j 0}\right|: j \in \mathbb{N}_{0}^{7}\right\} x$. Then, $\prod_{f}$ and $\prod_{I}$ are bi-non-increasing functions. The following assumptions hold: (H1) there exist non-negative bi-non-decreasing functions $\prod_{f}, \prod_{j}:[0, \infty) \times[0, \infty) \rightarrow[0, \infty)$, such that

$$
|f(t, x, y)| \leq \prod_{f}(|x|,|y|) t^{p}(1-t)^{q}, \quad\left|I_{j}\left(t_{s}, x, y\right)\right| \leq \prod_{I}(|x|,|y|) .
$$

(H2) there exists a constant $M>0$, such that for $x \in X$ with $|x(t)|>M$ for all $t \in(0,1]$ implies that

$$
\begin{aligned}
& \int_{0}^{1} \frac{(1-t)^{\alpha-n}}{\Gamma(\alpha-n+1)} f\left(t, x(t),{ }^{c} D_{0^{+}}^{\beta} x(t)\right) \mathrm{d} t+\sum_{w=1}^{m} I_{n-1}\left(t_{w}, x\left(t_{w}\right),{ }^{c} D_{0^{+}}^{\beta} x\left(t_{s}\right)\right) \\
& =\int_{0}^{1} \frac{(1-t)^{\alpha-n}}{\Gamma(\alpha-n+1)} t^{-\frac{1}{5}}(1-t)^{-\frac{1}{5}}\left(c_{0}+b_{0} x(t)+a_{0} \arctan \left({ }^{c} D_{0^{+}}^{\frac{1}{5}} x(t)\right)\right) \mathrm{d} t+B_{70} x(1 / 2) \\
& =c_{0} \frac{\mathbf{B}(2 / 5,4 / 5)}{\Gamma(3 / 5)}+b_{0} \int_{0}^{1} \frac{(1-t)^{-3 / 5}}{\Gamma(3 / 5)} t^{-\frac{1}{5}} x(t) \mathrm{d} t \\
& \quad+a_{0} \int_{0}^{1} \frac{(1-t)^{\alpha-n}}{\Gamma(\alpha-n+1)} t^{-\frac{1}{5}}(1-t)^{-\frac{1}{5}} \arctan \left({ }^{c} D_{0^{+}}^{\frac{1}{5}} x(t)\right) \mathrm{d} t+B_{70} x(1 / 2) .
\end{aligned}
$$

Since $B_{00}>-1$, we have $x\left(1 / 2^{+}\right)=\left(1+B_{00}\right) x\left(1 / 2^{-}\right)$. Therefore, either $x(t) \geq 0$ for all $t \in(0,1]$ or $x(t) \leq 0$ for all $t \in(0,1]$. Thus, there exists a constant $M>0$, such that $x \in D\left(L_{1}\right) \bigcap X$ with $|x(t)|>M$ implies

$$
\int_{0}^{1} \frac{(1-t)^{\alpha-n}}{\Gamma(\alpha-n+1)} f\left(t, x(t),{ }^{c} D_{0^{+}}^{\beta} x(t)\right) \mathrm{d} t+\sum_{w=1}^{m} I_{n-1}\left(t_{w}, x\left(t_{w}\right),{ }^{c} D_{0^{+}}^{\beta} x\left(t_{s}\right)\right) \neq 0 .
$$

(H3) there exists a constant $M_{0}>0$, such that

$$
\begin{aligned}
& c\left[\int_{0}^{1} \frac{(1-t)^{\alpha-n}}{\Gamma(\alpha-n+1)} f(t, c, 0) \mathrm{d} t+\sum_{w=1}^{m} I_{n-1}\left(t_{w}, c, 0\right]\right. \\
& =c\left[\int_{0}^{1} \frac{(1-s)^{\alpha-n}}{\Gamma(\alpha-n+1)} s^{-\frac{1}{5}}(1-s)^{-\frac{1}{5}}\left(c_{0}+c b_{0}\right) \mathrm{d} s+B_{7} 0 c\right] \\
& =\left(\frac{\mathbf{B}(2 / 5,4 / 5)}{\Gamma(3 / 5)} b_{0}+B_{70}\right) c^{2}+\frac{\mathbf{B}(2 / 5,4 / 5)}{\Gamma(3 / 5)} c_{0} c>0 \text { holds for all }|c|>M_{0}\left(\text { since } b_{0}+B_{7}>0\right) .
\end{aligned}
$$


By direct computation, we have

$$
\begin{aligned}
M_{1}= & 2 \sum_{v=1}^{7} \sum_{j=1}^{7} \frac{\Gamma(7)}{\Gamma(v+1)} \sum_{\sigma=7-j}^{7} \frac{(1 / 2)^{\sigma+j-7}}{\Gamma(\sigma+j-6)}+\sum_{v=1}^{7} \sum_{j=1}^{7} \frac{\Gamma(7)}{\Gamma(v+4 / 5)} \sum_{\sigma=7-j}^{7} \frac{(1 / 2)^{\sigma+j-7}}{\Gamma(\sigma+j-6)} \\
& +2 \sum_{v=0}^{7} \frac{1}{\Gamma(v+1)}+\sum_{v=0}^{7} \frac{1}{\Gamma(v+4 / 5)}, \\
M_{2}= & 2 \sum_{v=1}^{7} \sum_{j=1}^{7} \frac{\Gamma(7)}{\Gamma(v+1)} \frac{\mathbf{B}(2 / 5+j, 4 / 5)}{\Gamma(3 / 5+j)}+2 \frac{\mathbf{B}(37 / 5,4 / 5)}{\Gamma(38 / 5)}+\sum_{v=1}^{7} \sum_{j=1}^{7} \frac{\Gamma(7)}{\Gamma(v+4 / 5)} \frac{\mathbf{B}(2 / 5+j, 4 / 5)}{\Gamma(3 / 5+j)} \\
& +\frac{\mathbf{B}(36 / 5,4 / 5)}{\Gamma(37 / 5)} .
\end{aligned}
$$

Then, Theorem 3.1 implies that BVP (4.1) has at least one solution if

$$
\lim _{r \rightarrow \infty} \frac{r}{M+M_{1}\left[c_{0}+b_{0} r+a_{0} \arctan r\right]+M_{2} \max \left\{\left|B_{j 0}\right|: j \in \mathbb{N}_{0}^{7}\right\} r}=\frac{1}{M_{1} b_{0}+M_{2} \max \left\{\left|B_{j 0}\right|: j \in \mathbb{N}_{0}^{7}\right\}}>1 .
$$

Example 4.1 is completed.

Example 4.2 Let $y_{0}, y_{1}, I_{k}, J_{k} \in \mathbb{R}, 0<\xi_{1}<\cdots<\xi_{p}<1, b_{i} \geq 0$ with $\sum_{i=1}^{p} p_{i}<1, z \in C[0,1]$. Then

$$
\left\{\begin{array}{l}
{ }^{c} D_{0^{+}}^{q} y(t)+z(t)=0, t \in J^{\prime}=J\left\{t_{1}, t_{2}, \ldots, t_{p}\right\}, \quad J=[0,1], \\
\Delta y\left(t_{k}\right)=I_{k}, \Delta y^{\prime \prime}\left(t_{k}\right)=J_{k}, k=1, \ldots, p, \\
\Delta y^{\prime}\left(t_{k}\right)=0, k=1, \ldots, p \\
y^{\prime}(0)=0, y(0)=y_{0}, \quad y^{\prime}(1)=y_{1} .
\end{array}\right.
$$

Corresponding to (1.2), we find $g(y)=0, b_{i}=0, i \in \mathbb{N}_{1}^{p}$. According to Result 1 presented in Sect. 1 [14], we get the unique solution of (4.2) by

$$
y(t)=\left\{\begin{array}{l}
-\int_{0}^{t} \frac{(t-s)^{q-1}}{\Gamma(q)} z(s) \mathrm{d} s+y_{0}+\frac{c_{2}}{2} t^{2}, t \in J_{0}=\left[0, t_{1}\right], \\
-\int_{t_{1}}^{t} \frac{(t-s)^{q-1}}{\Gamma(q)} z(s) \mathrm{d} s-\int_{0}^{t_{1}} \frac{\left(t_{1}-s\right)^{q-1}}{\Gamma(q)} z(s) \mathrm{d} s-\frac{\left(t-t_{1}\right)^{2}}{2} \int_{0}^{t_{1}} \frac{\left(t_{1}-s\right)^{q-2}}{\Gamma(q-1)} z(s) \mathrm{d} s \\
+\frac{\left(t-t_{1}\right)^{2}}{2} J_{1}\left(y\left(t_{1}\right)\right)+I_{1}\left(y\left(t_{1}\right)\right)+y_{0}+\frac{c_{2}}{2}\left[t_{1}^{2}+\left(t-t_{1}\right)^{2}\right], t \in J_{1}=\left(t_{1}, t_{2}\right], \\
-\int_{t_{k}}^{t} \frac{(t-s)^{q-1}}{\Gamma(q)} z(s) \mathrm{d} s-\sum_{i=1}^{k} \int_{t_{i-1}}^{t_{i}} \frac{\left(t_{i}-s\right)^{q-1}}{\Gamma(q)} z(s) \mathrm{d} s-\frac{\left(t-t_{k}\right)^{2}}{2} \sum_{i=1}^{k} \int_{t_{i-1}}^{t_{i}} \frac{\left(t_{i}-s\right)^{q-3}}{\Gamma(q-2)} z(s) \mathrm{d} s \\
+\frac{1}{2} \sum_{i=2}^{k} \sum_{j=i-1}^{k-1}\left(t_{j+1}-t_{j}\right)^{2} \int_{t_{i-2}}^{t_{i-1}} \frac{\left(t_{i-1}-s\right)^{q-3}}{\Gamma(q-2)} z(s) \mathrm{d} s+\frac{\left(t-t_{k}\right)^{2}}{2} \sum i m i t s_{i=1}^{k} J_{i}\left(y\left(t_{i}\right)\right) \\
+\frac{1}{2} \sum_{i=1}^{k-1} \sum_{j=i}^{k-1}\left(t_{j+1}-t_{j}\right)^{2} J_{i}\left(y\left(t_{i}\right)\right)+\sum_{i=1}^{k} I_{i}\left(y\left(t_{i}\right)\right) \\
+y_{0}+\frac{c_{2}}{2}\left[\sum_{i=1}^{k}\left(t_{i}-t_{i-1}\right)^{2}+\left(t-t_{k}\right)^{2}\right], t \in J_{k}=\left(t_{k}, t_{k+1}\right], k=2,3, \ldots, p,
\end{array}\right.
$$

where

$$
c_{2}=-\int_{t_{p}}^{1} \frac{(1-s)^{q-3}}{\Gamma(q-2)} z(s) \mathrm{d} s+\sum_{i=1}^{p}-\int_{t_{i-1}}^{t_{i}} \frac{\left(t_{i}-s\right)^{q-3}}{\Gamma(q-2)} z(s) \mathrm{d} s-\sum_{i=1}^{p} J_{i}\left(y\left(t_{i}\right)\right)+y_{1} .
$$


According to Lemma $2.11,{ }^{c} D_{0^{+}}^{q} y(t)+z(t)=0, t \in\left(t_{i}, t_{i+1}\right], i \in \mathbb{N}_{0}^{p}$ implies that there exist constants $c_{i}, d_{i}, e_{i} \in \mathbb{R}$, such that

$$
y(t)=\sum_{j=0}^{i} c_{j}+\sum_{j=0}^{i} d_{j}\left(t-t_{j}\right)+\sum_{j=0}^{i} \frac{e_{i}}{2}\left(t-t_{j}\right)^{2}-\int_{0}^{t} \frac{(t-s)^{q-1}}{\Gamma(q)} z(s) \mathrm{d} s, \quad t \in\left(t_{i}, t_{i+1}\right], i \in \mathbb{N}_{0}^{p} .
$$

Then

$$
y^{\prime}(t)=\sum_{j=0}^{i} d_{j}+\sum_{j=0}^{i} e_{i}\left(t-t_{j}\right)-\int_{0}^{t} \frac{(t-s)^{q-2}}{\Gamma(q-1)} z(s) \mathrm{d} s, \quad t \in\left(t_{i}, t_{i+1}\right], i \in \mathbb{N}_{0}^{p},
$$

and

$$
y^{\prime \prime}(t)=\sum_{j=0}^{i} e_{j}-\int_{0}^{t} \frac{(t-s)^{q-1}}{\Gamma(q)} z(s) \mathrm{d} s, \quad t \in\left(t_{i}, t_{i+1}\right], i \in \mathbb{N}_{0}^{p} .
$$

Using $y^{\prime}(0)=0, y(0)=y_{0}$, and $y^{\prime \prime}(1)=y_{1}$, we get $c_{0}=y_{0}, d_{0}=0$, and

$$
\sum_{j=0}^{p} e_{j}-\int_{0}^{1} \frac{(1-s)^{q-3}}{\Gamma(q-2)} z(s) \mathrm{d} s=y_{1} .
$$

Using $\Delta y\left(t_{k}\right)=I_{k}, \Delta y^{\prime \prime}\left(t_{k}\right)=J_{k}$, and $\Delta y^{\prime}\left(t_{k}\right)=0$ for $k=1, \ldots, p$, we get $c_{k}=I_{k}, d_{k}=0$, and $e_{k}=J_{k}$ for $k \in \mathbb{N}_{1}^{p}$. Then

$$
e_{0}=-\sum_{j=1}^{p} J_{j}+\int_{0}^{1} \frac{(1-s)^{q-3}}{\Gamma(q-2)} z(s) \mathrm{d} s+y_{1} .
$$

Hence, the unique solution of (4.2) is given by

$$
\begin{aligned}
y(t)= & y_{0}+\sum_{j=1}^{i} I_{j}-\frac{1}{2}\left[y_{1}-\sum_{j=1}^{p} J_{j}+\int_{0}^{1} \frac{(1-s)^{q-3}}{\Gamma(q-2)} z(s) \mathrm{d} s\right] t^{2}+\sum_{j=1}^{i} \frac{J_{j}}{2}\left(t-t_{j}\right)^{2} \\
& -\int_{0}^{t} \frac{(t-s)^{q-1}}{\Gamma(q)} z(s) \mathrm{d} s . \quad t \in\left(t_{i}, t_{i+1}\right], i \in \mathbb{N}_{0}^{p} .
\end{aligned}
$$

One sees that (4.4) is not equivalent to (4.3).

Acknowledgments Supported by the Natural Science Foundation of Guangdong province (No: S2011010001900) and Natural science research project for colleges and universities of Guangdong Province (No: 2014KTSCX126).

Open Access This article is distributed under the terms of the Creative Commons Attribution 4.0 International License (http:// creativecommons.org/licenses/by/4.0/), which permits unrestricted use, distribution, and reproduction in any medium, provided you give appropriate credit to the original author(s) and the source, provide a link to the Creative Commons license, and indicate if changes were made.

\section{References}

1. Hilfer, R.: Applications of Fractional Calculus in Physics. World Scientific, River Edge (2000)

2. Kilbas, A.A.; Srivastava, H.M.; Trujillo J.J.: Theory and Applications of Fractional Differential Equations. Elsevier, Amsterdam (2006)

3. Lakshmikantham, V.V.; Bainov D.D.; Simeonov, P.S.: Theory of Impulsive Differential Equations. World Scientific, Singapore (1989)

4. Lashmikanthan, V.; Vatsala, A.S.: Basic theory of fractional differential equations. Nonlinear Anal. 69, 2715-2682 (2008)

5. Liu, G.; Wu, T.: Differential quadrature solutions of eighth-order boundary-value differential equations. J. Comput. Appl. Math. 145, 223-235 (2002)

6. Mawhin, J.: Topological degree methods in nonlinear boundary value problems. In: CBMS Regional Conference Series in Mathematics 40. American Math. Soc. Providence (1979) 
7. Miller, K.S.; Ross B.: An Introduction to the Fractional Calculus and Fractional Differential Equations. John Wiley, New York (1993)

8. Nyamoradia, N.; Rodriguez-Lopez, R.: On boundary value problems for impulsive fractional differential equations. Appl. Math. Comput. 271(C), 874-892 (2015)

9. Podlubny, I.: Fractional Differential Equations. Mathmatics in Science and Engineering, vol. 198. Academic Press, San Diego (1999)

10. ur Rehman, M.; Eloe, P.W.: Existence and uniqueness of solutions for impulsive fractional differential equations. Appl. Math. Comput. 224, 422-431 (2013)

11. Wang, H.; Lin, X.: Anti-periodic BVP of fractional order with fractional impulsive conditions and variable parameter. J. Appl. Math. Comput. (2015). doi:10.1007/s12190-015-0968-5

12. Wang, J.; Zhang, Y.: On the concept and existence of solutions for fractional impulsive systems with Hadamard derivatives. Appl. Math. Lett. 39, 85-90 (2015)

13. Wu, T.; Liu, G.: Application of the generalized differential quadrature rule to eighth-order differential equations. Commun. Numer. Methods Eng. 17(5), 355-364 (2001)

14. Yang, J.; Liu, D.; Zhang, B., et al.: Existence of solutions for high-order impulsive boundary value problem of quasilinear fractional differential equation. Acta Scientiarum Naturalium Universitatis Sunyatseni. 53(1), 34-41 (2014)

15. Yukunthorn, W.; Ahmad, B.; Ntouyas, S.K.; Tariboon, J.: On CaputoCHadamard type fractional impulsive hybrid systems with nonlinear fractional integral conditions. Nonlinear Anal. Hybrid Syst. 19, 77-92 (2016)

16. Zhang, X.: On the concept of general solution for impulsive differential equations of fractional order $q \in(1,2)$. Appl. Math. Comput. 268, 103-120 (2015)

17. Zhang, X.; Zhu, C.; Wu, Z.: Solvability for a coupled system of fractional differential equations with impulses at resonance. Bound. Value Probl. 80, 14 pages (2013)

18. Zhao, K.; Gong P.: Positive solutions for impulsive fractional differential equations with generalized periodic boundary value conditions. Adv. Differ. Equ. 2014, 255 (2014). doi:10.1186/1687-1847-2014-255 